\title{
In Utero Exposure to Simulated Complex Urban Air Pollution Disturbs Intestinal Suckling-to-Weaning Transition in Mice
}

\section{Eva Guilloteau}

University of Lille: Universite de Lille

\section{Patrice COLL}

LISA: Laboratoire Inter-universitaire des Systemes Atmospheriques

\section{Zhuyi LU}

University Paris-Est Créteil Val de Marne: Universite Paris-Est Creteil Val de Marne

\section{Madjid DJOUINA}

University of Lille: Universite de Lille

\section{Mathieu CAZAUNAU}

LISA: Laboratoire Inter-universitaire des Systemes Atmospheriques

\section{Christophe WAXIN}

University of Lille: Universite de Lille

\section{Antonin BERGE}

LISA: Laboratoire Inter-universitaire des Systemes Atmospheriques

\section{Ségolène CABOCHE}

University of Lille: Universite de Lille

\section{Aline GRATIEN}

LISA: Laboratoire Inter-universitaire des Systemes Atmospheriques

\section{David HOT}

University of Lille: Universite de Lille

\section{Laurent DUBUQUOY}

University of Lille: Universite de Lille

\section{David LAUNAY}

University of Lille: Universite de Lille

\section{Cécile VIGNAL}

University of Lille: Universite de Lille

\section{Sophie LANONE}

University Paris-Est Créteil Val de Marne: Universite Paris-Est Creteil Val de Marne

Mathilde Body-Malapel ( $\nabla$ mathilde.body@univ-lille.fr )

Universite de Lille https://orcid.org/0000-0003-3392-4229 
Research

Keywords: Air pollution, Gestational exposure, Intestinal development, Suckling-to-weaning transition, Microbiota, Maturation, Inflammation, Sex-specific,

Posted Date: November 11th, 2021

DOI: https://doi.org/10.21203/rs.3.rs-1040168/v1

License: (c) (i) This work is licensed under a Creative Commons Attribution 4.0 International License.

Read Full License 


\section{Abstract \\ Background}

Emerging data indicate that prenatal exposure to air pollution may lead to higher susceptibility to several non-communicable diseases. Limited research has been conducted due to difficulties in modelling realistic air pollution exposure. In this study, pregnant mice were exposed from gestational day 10 to 17 to an atmosphere representative of a 2013 pollution event in Beijing, China. Intestinal homeostasis and microbiota were assessed in both male and female offspring during the suckling-to-weaning transition.

\section{Results}

Sex-specific differences were observed in progeny of gestationally-exposed mice. In utero exposed males exhibited decreased villus and crypt length, vacuolation abnormalities, and lower levels of tight junction protein ZO-1 in ileum. They showed an upregulation of absorptive cell markers and a downregulation of neonatal markers in colon. Cecum of in utero exposed male mice also presented a deeply unbalanced inflammatory pattern. By contrast, in utero exposed female mice displayed less severe intestinal alterations, but included dysregulated expression of Lgr5 in colon, Tjp1 in cecum, and Epcam, Car2 and Sis in ileum. Moreover, exposed female mice showed dysbiosis characterized by a decreased weighted UniFrac $\beta$-diversity index, a higher abundance of Bacteroidales and Coriobacteriales orders, and a reduced Firmicutes/Bacteroidetes ratio.

\section{Conclusion}

Prenatal realistic modelling of an urban air pollution event induced sex-specific precocious alterations of structural and immune intestinal development in mice.

\section{Background}

Air pollution has been estimated to be the single most important environmental health risk factor. It represents a greater disease burden than polluted water, soil contamination, and occupational exposures combined [1]. Air pollution causes a loss of life expectancy which rivals that of tobacco smoking [2]. It is well recognized as a major risk factor for many chronic non-communicable diseases such as cardiovascular, pulmonary and metabolic diseases [3-5]. Association studies also suggest a possible contribution of air pollution to the development of neurological illnesses such as Alzheimer's disease [6], chronic kidney disease and renal function decline [7], liver cirrhosis [8], and autoimmune diseases. For the latter, epidemiological studies have shown a relationship between exposure to air pollution and development and progression of multiple sclerosis [9], and exacerbation of rheumatoid disease [10] and systemic lupus erythematosus $[11,12]$. Furthermore, air pollution may also contribute to intestinal diseases $[13,14]$. 
There is a growing body of evidence which indicates that there is a prenatal window of susceptibility to adverse effects of air pollution. Exposure to air pollution early in life is directly linked to the development of major cardiovascular risks, including obesity, hypertension, and metabolic disorders $[15,16]$. Air pollution exposure in utero has been associated with childhood asthma and allergic diseases $[17,18]$. Regarding the intestinal tract, a population-based epidemiological study has highlighted that Ox exposure (as measured by redox-weighted oxidant capacity, a measure that takes into account the oxidative potential of both ozone $\left(\mathrm{O}_{3}\right)$ and $\mathrm{NO}_{2}$ ) during the second trimester of pregnancy is associated with inflammatory bowel disease (IBD) development [19]. In mice, $\mathrm{PM}_{2.5}$ exposure during gestation caused changes in the distribution and structure of gut microbiota of dams [20]. However, despite the emerging role of air pollution in intestinal pathologies, animal studies focusing on the intestinal burden induced by in utero exposure to air pollution are missing. Moreover, despite ambient air pollution consisting of both particulate matter (PM) and gaseous components including $\mathrm{O}_{3}$, volatile organic compounds (VOCs), carbon monoxide (CO), and nitrogen oxides (NOx), realistic experimental studies that simulate complex air pollution are lacking. In this work, we aimed to assess the early effects of gestational exposure to complex urban air pollution on intestinal tissues. For this purpose a more representative atmospheric model, based on a 2013 pollution event in Beijing, was generated. Pregnant mice were exposed to simulated Beijing-like air pollution from gestational day 10 (GD10) to GD17. Control mice were exposed to filtered Beijing-like air pollution during the same period. Effects of intrauterine exposure were assessed during the suckling-to-weaning transition (at postnatal day 17), which corresponds to a critical window for both structural and immune intestinal development [21, 22].

\section{Results}

\section{Histomorphological analysis in male mice}

In mice, as in humans, the neonatal intestine has several features that are distinct from adults. During the suckling-to-weaning period, gut growth and maturation accelerate involving both functional and structural changes in gut epithelium [23]. Thus, we first performed an overall assessment of the gut mucosal structure. May Grünwald Giemsa (MGG)-stained sections of male colon and ileum were analyzed (Fig $1 \mathrm{~A}$ and $\mathrm{D}$ ). In colon, the cell number in the submucosa and the mucosal surface area were measured (Fig 1 B and C). No variation was evident. In ileum, the villus length and the crypt depth were measured (Fig $1 \mathrm{E}$ and $\mathrm{F}$ ). They were both significantly decreased in Beijing-like air mice compared to control air mice. The villus/crypt ratio did not vary (Fig 1G). Furthermore, during the suckling-to-weaning period, vacuolated fetal-type epithelium was replaced by non-vacuolated adult-type epithelium [23]. Since the small intestine of GD17 pups presented intense vacuolation of the villi, we developed a method for quantitative assessment of this vacuolation. The number of vacuoles per villi was determined and did not show variation between the 2 groups of mice $(\mathrm{Fig} 1 \mathrm{H})$. The mean vacuolated area per villus was significantly reduced in Beijing-like air mice (Fig 1I). Morphometric evaluation of the vacuoles also showed significant variations (Fig $1 \mathrm{~J}$ and $\mathrm{K}$ ). In pups exposed in utero to air pollution, the vacuole circularity index was increased and the mean eccentricity index was decreased compared to control pups. 
Therefore, in males, postnatal exposure to air pollution induced histomorphological alterations in ileum, and notable disturbances of the vacuolation process.

\section{Histomorphological analysis in female mice}

We performed the same analyses in colon and ileum of female pups. In colon, the mucosal surface area and the submucosa cellularity did not show significant variation between air pollution- and controlexposed mice (Fig 2A, B, C). In ileum, there were no modification of the crypt depth, villus length, and villus/crypt ratio (Fig 2D, E, F, G). The morphometric analyses of the vacuoles showed no differences between the female pups in utero exposed to simulated Beijing-like polluted air compared to female control pups. Therefore, in females, no important histological impairments of colon and ileum were induced by the postnatal exposure of simulated Beijing-like air pollution.

\section{Epithelium proliferation, differentiation, and maturation in male mice}

To further examine epithelium proliferation, immunohistochemical staining of the proliferative marker PCNA (proliferating cell nuclear antigen) was performed. In male colon, the number of PCNA-positive cells was counted and was similar between the polluted air- and the control air-exposed mice (Fig $3 \mathrm{~A}$ and B). Transcript levels of several markers of intestinal cells were quantified by real-time polymerase chain reaction (PCR). The leucine-rich-repeat-containing G-protein-coupled receptor $5(L g r 5)$, the best described intestinal stem cell marker, did not show significant variation (Fig $3 C$ ). The markers of absorptive cells alkaline phosphatase, intestinal (Alpi), epithelial cell adhesion molecule (Epcam), and carbonic anhydrase 2 (Car2) were all significantly upregulated in Beijing-like air mice compared to control air mice (Fig 3D). The marker of goblet cells mucin 2 (Muc2) was significantly downregulated in Beijing-like air mice (Fig $3 \mathrm{E})$. The transcript levels of the markers of Paneth cells (lysozyme $1 ; L y z 1$ ), of enterochromaffin cells (chromogranin A; ChrgA), and of tuft cells (POU domain, class 2, transcription factor 3; Pou2f3), were not changed between the 2 groups of mice.

To assess the functional maturation of gut epithelium, we quantified mRNA levels of several neonatal and adult markers (Fig $3 \mathrm{~F}$ and $\mathrm{G}$ ) [22]. During the suckling period, neonatal intestinal cells express disaccharidase lactase-phlorizin hydrolase ( $L C t)$ to digest milk lactose [1]. After the suckling period, enterocytes adapt to digest solid food that is rich in complex carbohydrates and low in fat. This is manifested by a switch in brush border disaccharidase expression from lactase to sucrase isomaltase (Sis) and trehalase (Treh) [2]. In colon of Beijing-like air mice compared to control air mice, expression of Lctwas downregulated, expression of Treh was unchanged, and expression of Sis was upregulated. Furthermore, another metabolic switch is related to the low concentration of arginine in milk. To provide for the need for arginine, neonatal enterocytes express the rate limiting enzyme in arginine biosynthesis, argininosuccinate synthase-1 (Ass1) [3]. In contrast, adult enterocytes express arginase 2 (Arg2), an enzyme capable of catabolizing arginine that is abundant in solid foods. Colon expression of Ass 1 and Arg2 were lower in mice exposed in utero to Beijing-like air pollution as compared with mice exposed to control air pollution. Furthermore, the neonatal intestinal epithelium expresses the neonatal Fc fragment of the IgG receptor and transporter ( $F c g r t$, also called $F c R n$ ), which mediates absorption of maternal IgG 
from milk into the bloodstream [24]. The expression of Fcgrt declines significantly during the suckling-toweaning transition [22]. Similarly, the expression of Prdm1 (PR domain containing 1, with ZNF domain; also called Blimp-1, or B lymphocyte-induced maturation protein-1) is lost at the suckling-to-weaning transition [25,26]. Prdm1 expression was not modified but Fcgrt transcript levels were significantly reduced in colon after Beijing-like air pollution exposure.

In ileum, the number of PCNA-positive cells was higher in Beijing-like air mice compared to control air mice, reflecting an increase of epithelial proliferation in these mice (Fig $3 \mathrm{H}$ and I). The mRNA levels of the markers of stem, absorptive, and secretory cells were not modified (Fig $3 \mathrm{~J}-\mathrm{L}$ ). Moreover, there was no variation of expression of the neonatal and adult genes (Fig $3 \mathrm{M}$ and $\mathrm{N}$ ).

Altogether, these results tend to show that postnatal exposure to simulated Beijing-like air pollution in male pups led to alterations of differentiation and maturation biomarkers in colon epithelial cells, as well as to an increase of epithelial proliferation in ileum.

\section{Epithelium proliferation, differentiation, and maturation in female mice}

In females, PCNA immunostaining of colon showed no significant modification of proliferation in Beijinglike air mice compared to control air mice (Fig $4 \mathrm{~A}$ and B). The mRNA level of $L$ gr5 was significantly downregulated in Beijing-like air mice (Fig 4C). The transcript abundance of absorptive and secretory cell markers did not show significant variation (Fig $4 \mathrm{D}$ and $\mathrm{E}$ ). The neonatal markers were not changed either (Fig 4F). The transcription of Arg2 was significantly downregulated in Beijing-like air mice compared to control air mice (Fig 4G).

In ileum, PCNA staining was similar between the Beijing-like air mice and the control air mice (Fig $4 \mathrm{H}$ and I). The level of $L g r 5$ was also unchanged (Fig $4 \mathrm{~J}$ ). The expression of absorptive cell markers Epcam and Car2 were upregulated in Beijing-like air mice (Fig 4K). Among the secretory cell markers, a relative overexpression of ChrgA transcripts was quantified in Beijing-like air mice as compared with control air mice (Fig 4L). The maturation of ileal tissue was weakly altered, and an upregulation of Sis expression was detected in Beijing-like air mice (Fig $4 \mathrm{M}$ and N). Because over-expression of ChgrA in Beijing-like airexposed mice ileum was observed, we quantified expression of other markers of enteroendocrine cells. Levels of tachykinin 1 (Tac1, or substance P)-producing enterochromaffin cells, glucagon (Gcg)- and peptide YY (Pyy)-expressing L cells, gastric inhibitory protein (Gip)-producing K cells, neurotensin (Nts)and neuromedin-producing $\mathrm{N}$ cells, and secretin (Sct)-producing S cells were quantified [27] (Fig S2). An upregulation of Nts was found in the Beijing-like air-polluted group, reflecting an abnormal expression of neurotensin.

\section{Inflammatory pattern in male mice}

In order to assess whether in utero exposure to air pollution induces impairments of the inflammatory process in GD17 pups, we quantified mRNA levels of transcription factors and inflammatory cytokines representative of the Th1, Th2, Th17, and Treg immune response. In proximal colon, expression of the 
immunomodulatory cytokine //10 was strongly enhanced in Beijing-like air pups compared to control air pups (Fig 5A). In cecum, the Th1 transcription factor Tbx21 (also called T-bet) was significantly higher in Beijing-like air mice compared to control mice (Fig 5B). The Th2 cytokines //4 and //5 were also upregulated in Beijing-like air pups. The Th17 transcription factor Rorc was upregulated and the levels of I/17a and I/22 were downregulated in Beijing-like air mice compared to control air mice. Levels of Tgfb were also lower in cecum of Beijing-like air mice. In ileum, a significant upregulation of Rorc was induced by in utero exposure of simulated Beijing-like air pollution (Fig 5C).

\section{Inflammatory pattern in female mice}

In proximal colon of female mice, the transcript levels of $/ / 13$ and $/ / 10$ cytokines were greatly upregulated in Beijing-like air mice compared to control air mice (Fig 6A). In addition, the mRNA expression of $/ / 4$ was enhanced in cecum by in utero Beijing-like air exposure (Fig 6B). None of the other markers studied were modified in ileum (Fig 6C).

\section{Intestinal permeability markers in male and female mice}

We assessed whether in utero exposure to air pollution disrupts the intestinal barrier. The transcript levels of 3 markers of intestinal permeability, occludin (OCln), tight junction protein-1 (Tjp 1; coding forzonula occludin 1 protein), and claudin-4 (Cldn4), were quantified. In male proximal colon there was no significant expression variation of these 3 markers (Fig 7A). In cecum of Beijing-like air-exposed males, we observed an upregulation of Ocln and a downregulation of Tjp1 and Cldn4 transcripts. Tjp1 expression was also strongly reduced in the ileum of males exposed to Beijing-like air. In females, in utero Beijing-like air pollution exposure induced an upregulation of Cldn4 and Tjp 1 in proximal colon and cecum, respectively (Fig 7B). Furthermore, we quantified the expression of ZO-1 protein by western blot and confirmed that ZO-1 protein levels were significantly reduced in ileum of male mice exposed in utero to Beijing-like air pollution compared to control air mice (Fig $7 \mathrm{C}$ and $\mathrm{D}$ ).

\section{Microbiota DNA extraction and 16S rDNA gene amplicon sequencing analysis}

To assess the impact of in utero exposure to air pollution on colon luminal bacterial content, we sequenced V3-V4 amplicons of 16S rRNA genes. After a denoising step performed with DADA2 software, we obtained a total of 884,707 reads. Exposure to air pollution did not significantly affect a-diversity (Chao1 diversity index, Fig 8A; Evenness and Simpson indices, Fig S3). The weighted UniFrac b-diversity index showed no significant difference in males, but a significant decrease in females ( $p=0.011$; Fig 8B). After taxonomic assignment of amplicon sequence variants (ASVs), the effect of in utero air pollution exposure on the abundance of phyla was assessed. Taxonomic assignment at the phylum level of ASVs, with each color representing an individual bacterial phylum, is shown in Fig 8C. Bacterial composition was dominated by members of the Bacteroidetes phylum followed by Firmicutes, although mice presented important interindividual variations. A high abundance of Verrucomicrobia was found in only 4 control male and 2 control female mice, but no phylum was found to be significantly different between the exposed and control mice. Similarly, analyses at the class level showed important interindividual 
variability, but did not reveal significant variations between air pollution- and control-exposed groups (data not shown). At the order level, the main bacteria were Bacteroidales,

Clostridiales, and Lactobacillales (Fig 8D). Bacteroidales and Coriobacteriales orders were significantly

more abundant in Beijing-like air-exposed mice than in control air-exposed female mice $(p=0.001$ and $\mathrm{p}=0.04$, respectively; Fig $8 \mathrm{E}$ ). Moreover, the Firmicutes/Bacteroides ratio, which is a widely used marker of intestinal dysbiosis, was calculated and found to be significantly reduced in females after in utero exposure to Beijing-like air (Fig 8F).

\section{Discussion}

Although exposure to air pollution during pregnancy is linked to high risk of adverse pregnancy outcomes and long-term postnatal health, limited mechanistic data exists to assess these impacts under controlled exposure conditions. This limitation of our knowledge is mainly attributed to the complexity of the polluted atmosphere, and to the great difficulty in modelling the impact of realistic exposures. Using CESAM, an atmospheric simulation chamber, we have capitalized on a totally innovative platform for exposing mice to more realistic atmospheric conditions. Among the constituents of air pollution associated with deleterious effects on health, we considered gaseous pollutants $\mathrm{O}_{3}, \mathrm{SO}_{2}, \mathrm{CO}$, NOx, and VOCs) and particles ( $\mathrm{PM}_{10}, \mathrm{PM}_{2.5}$, and ultrafine $\left.\mathrm{PM}\right)$. In order to simulate atmospheric mixtures in all their complexity in the laboratory, environmental chemists have developed photo-reactors that are equipped to reproduce and control atmospheric processes such as solar radiation, concentrations of species, and the timely injection of aerosols and gases. These atmospheric simulation chambers thus offer the possibility of studying the myriad of products resulting from the atmospheric oxidation of primary compounds [28].

This innovative experimental approach allowed us to mimic the effects of "real life" exposure of urban air pollution on the suckling-to-weaning intestinal tissues. In mice intestinal development begins right before birth and intestinal maturation is completed at approximately 3 weeks postnatally during the suckling-toweaning transition [29]. In female offspring, in utero exposure to Beijing-like air pollution induced mild modifications of intestinal development. Enhanced expression of Epcam and Car2 absorptive epithelial cell markers and ChrgA enteroendocrine cell marker were observed in small intestine. In colon, the only alterations were decreased expression of $L g r 5$ stem cell marker and $A r g 2$ adult epithelial cell marker. Therefore, our results argue in favor of weak effects of postnatal air pollution exposure on intestinal differentiation and maturation in female young offspring. By contrast, in males, several parameters of intestinal development were affected by air pollution exposure. Notably, in small intestine, both the villus length and the crypt depth were reduced. This was associated with increased epithelial proliferation, as well as decreased size and morphological alterations of the vacuoles. This latter observation may reflect a trend to replacement of vacuolated fetal-type epithelium with non-vacuolated adult-type epithelium and therefore more precocious maturation in in utero pollution-exposed mice. However, the markers of neonatal cells were not changed, indicating the absence of a functional impact of exposure to air pollution on intestinal maturation. Moreover, our results show that in utero exposure to Beijing-like air pollution led to disturbances of proliferation processes. It is known that villus and crypt morphogenesis 
occurs during embryogenesis and postnatally, respectively; crypts are the architectural unit of the stem cell niche [30]. Villus and crypt morphogenesis are complex processes which are essential for normal intestinal physiology. A precedent for imprinting of skin epithelial stem cells has been reported [31]. Therefore, the abnormalities observed in male small intestine may reflect disorders of intestinal development which deserve further study.

We also studied whether in utero air pollution exposure could affect the maturation of the immune system. The expression of the immunomodulatory cytokine IL-10 was exacerbated in colon by in utero exposure to Beijing-like air, both in males and females. In neonates, as in adults, IL-10 can have antiinflammatory properties and can be produced by macrophages and T cells [32]. IL-10R signaling in macrophages is pivotal in confining a microbiota-driven inflammatory response beginning at the third week of life [33]. IL-10 can also be produced by neonatal type 1 conventional dendritic cells (CDC1) before their differentiation into IL-12p40-producing cDC1 [34]. Therefore, if IL-10 overexpression derives from $\mathrm{CDC1}$, it could reflect a defect in maturation of these cells. Apart from this finding, the cytokine profile in the colon was not altered. In ileum, the cytokine pattern was very similar between Beijing-like air mice and control air exposed mice. By contrast, in cecum, and only in males, we observed an increase of 2 major immune transcription factors (Tbx21 and Rorc), an overexpression of Th2 cytokines (I/4 and //5), and decreased expression of cytokines $/ 177 a, I / 22$, and $\operatorname{Tgf} \beta$. These alterations are numerous but they do not make it possible to demonstrate a clear defect in the immune response. Association of the upregulation of Th17 transcription factor Rorc and downregulation of Th17 cytokines I/17a and I/22 is paradoxical, and to date the data in the literature is not sufficient to fully explain this apparent discrepancy. It is known that immune homeostasis in the gut is normally maintained by the production of low levels of IL-17A and IL-22 by resident Th17 lymphocytes [35] and ILC3 [36]. In neonates IL-17A can also be produced by yठT cells and has an essential role in host defense against $C$. difficile infection [37].

Another important parameter of intestinal immune response is the development of an efficient mucosal barrier including a tailored regulation of tight junctions which seal the epithelial cell-cell contacts and regulate the paracellular passage of solutes [38]. The modifications of permeability markers that we observed, particularly downregulation of ZO-1 at the transcript and protein levels in male ileum, could lead to a gut barrier defect. The leakiness of the gut epithelium has been associated with the development of allergic and autoimmune diseases, especially when it is associated with a dysbiotic microbiota that cross the damaged barrier [39].

From birth, the normal gut microbiota contributes to the development of gut function, educates the immune system, contributes to the regulation and maintenance of intestinal barrier function, provides protection against infection, and promotes tolerance of foods. In addition, the early life microbiota has a crucial role in the risk of acquiring diseases such as asthma, atopic dermatitis, diabetes, allergic diseases, obesity, cardiovascular diseases, and neurological disorders [40]. In our study, in utero exposure to air pollution did not modify the a-diversity (within sample diversity) and especially the Chao 1 species richness index, indicating that the number of different species was similar between the groups. By contrast, and only in females, the bacterial composition showed few significant variations. At the phylum 
level, the ratio of Firmicutes/Bacteroidetes was reduced. In adults, this ratio is a hallmark of low-grade inflammation [41]. It has also been proposed as a marker of intestinal maturation during aging (in the second year of adulthood) [42]. In female mice exposed in utero to air pollution, the relative abundance of Coriobacteriales was higher. This order is reduced in colitic mice [43] and enriched in acute liver failure [44]. By contrast, Coriobacteriales are more abundant in diseased mucosal ileum tissues from Crohn's disease patients compared to control mucosa from non-IBD patients [45]. Coriobacteriales are also more abundant in fecal microbiota of multiple sclerosis patients [46]. Moreover, female mice exposed in utero to air pollution presented higher abundance of Bacteroidales. The Bacteroidales (or S24-7 family) are numerically dominant intestinal organisms that associate with the mucosal surface and have properties that both positively and negatively affect the host. The Bacteroidales showed a higher abundance in dextran sulfate sodium-induced mice $[43,47]$. In adult mice, bacteria from the order Bacteroidales are sufficient to promote appearance of intraepithelial lymphocytes in the colon, which are important for the maintenance of a healthy intestinal barrier [48]. In humans, Bacteroidales produce bacteroidetocins, a family of broad-spectrum peptide toxins that kill members of the Bacteroidetes phylum, including Bacteroides, Parabacteroides, and Prevotella gut species, as well as pathogenic Prevotella species [49]. A decrease in adherent Bacteroidales diversity (i.e., the number of different Bacteroides species per biopsy) has been found at sites with increased inflammation in IBD subjects [50]. Therefore, current knowledge shows that bacteria of the orders Bacteroidales and Coriobacteriales have a role in the regulation of intestinal homeostasis and are deregulated in certain pathological conditions, but this does not allow us to predict the consequences of their deregulation in females having been exposed in utero to air pollution.

Most of the effects that we observed in the gut as consequences of maternal air pollution exposure are sex-specific. This gender dependency is a classical feature of air pollution impact. For instance, prenatal air pollution exposure has been shown to "program" offspring for increased susceptibility to diet-induced weight gain and neuroinflammation in adulthood in a sex-specific manner [51]. Moreover, the effects induced on the immune system of offspring by maternal exposure to diesel exhaust particles (DEP) are also sex-dependent. Indeed, exposure to DEP in utero decreased the frequency of $C D 1 d^{\text {high }} \mathrm{CD}^{+} \mathrm{B}$ cells in female mice and IFN-y production by splenocytes in both sexes. Male mice had elevations in macrophage and lymphocyte numbers in response to DEP whereas female mice only had elevated IL-6, MCP-1, and MIP-2 levels [52]. Epidemiological studies have also highlighted gender-specific effects of air pollution on respiratory health. Overall, studies of children suggest stronger effects among boys in early life and among girls in later childhood [53].

Despite accumulating evidence of sex-dependent adverse effects of maternal air pollution exposure, the mechanisms involved are only now starting to be identified. Firstly, prenatal air pollution exposure induces epigenetic modifications in placenta and in cord blood. Notably, ambient PM causes significant epigenomic changes, including alterations in DNA methylation, miRNA regulation, and histone modifications. Birth cohort studies have shown that $\mathrm{PM}_{2.5}$ exposure during the last trimester of pregnancy was positively associated with placental methylation of the promoter regions of regulatory genes in the circadian pathway and key DNA repair genes [54]. $\mathrm{PM}_{10}$ exposure during the first 2 trimesters 
of pregnancy was positively associated with placental methylation of HSD11B2 (i.e., genes involved in the glucocorticoid metabolism and fetal growth). Moreover, specific $\mathrm{PM}_{2.5}$ pollution exposure windows were associated with altered placental miR-20a, miR-21, miR-146a, and miR-222 expression [55]. Also, prenatal $\mathrm{PM}_{2.5}$ exposure was positively associated with cord plasma histone $\mathrm{H} 3$ modifications [56]. This altered biomolecular functioning of the placenta may contribute to early and even later-life health consequences.

\section{Conclusions}

In mice, postnatal exposure to simulated Beijing-like air pollution induced sex-specific effects on intestinal proliferation, maturation, permeability, immune response, and microbiota composition. According to the "Developmental Origins of Health and Disease" (DOHaD) concept, early-life alterations induced by intrauterine pollutant exposure may have long-term effects influencing offspring susceptibility to diseases later in adulthood. Therefore, precocious disturbances that we observed in intestinal development following prenatal exposure to air pollution may underlie a higher susceptibility for developing diseases later in life.

\section{Methods}

\section{Generation of complex air pollution}

The innovative approach developed for this study was to realistically simulate the atmospheric mixture in its whole complexity, while maintaining the ability to control, reproduce, and fully characterize the experimental conditions. The CESAM chamber (described at https://cesam.cnrs.fr/; a $4.2 \mathrm{~m}^{3}$ stainless steel atmospheric simulation chamber; evaceable down to a few $10^{-7}$ atm; temperature controlled between $+15^{\circ} \mathrm{C}$ and $+60^{\circ} \mathrm{C}$ ) was used to study the myriad of products arising from the atmospheric oxidation of primary organic compounds. The experimental protocol included a continuous injection of relevant mixtures of primary pollutants (mainly nitrogen oxides, organic compounds from a representative mix of anthropogenic emissions, sulphur dioxide, soot, inorganic salts, and mineral dust particles from the Gobi Desert to simulate a Beijing-like atmosphere from 2013) at low concentrations (ppb levels) in air with the CESAM simulation chamber operating as a slow flow reactor. The residence time of simulated air parcels in the experimental volume was fixed to $4 \mathrm{~h}$ in order to represent air masses of regional scale. During this time the synthetic mixture was exposed to artificial solar irradiation, allowing secondary pollutants such as $\mathrm{O}_{3}$, nitric acid, formaldehyde, peroxyacetyl nitrates (PANs) as well as complex polyfunctional organics including secondary organic aerosols, to be produced and to reach their chemical steady state. Mice were exposed to constant flows of this mixture.

\section{Simulated atmospheric environment at the laboratory}

The 2013 China smog event was an extreme air pollution episode that affected East China, initiated by slow air carrying industrial emissions and meteorological conditions, that took place in December of that 
year. High levels of $\mathrm{PM}_{2.5}$ (generated from coal combustion and industrial sources) and low visibility were observed, leading to the closure of airports, highways, and schools. For the simulation of a Beijing-like atmosphere, VOCs were introduced into the atmospheric chamber as precursors, which were the most abundant and commonly emitted particulates in the urban atmosphere of Beijing at the time: isopentane (alkane), propene (alkene), acetylene (alkyne), acetaldehyde (aldehyde), benzene, toluene, and m,p-xylene (aromatic hydrocarbons). The concentrations were determined in order to have stable levels of pollutants in the chamber, and representative of the pollution episode [28]. By the end of $20 \mathrm{~h}$ of VOC injection, nitrogen monoxide (NO) was introduced in the chamber and then the lights were turned on, simulating the sunlight irradiation in the troposphere. Additionally, seeds of ammonium sulfate particles were injected in the chamber. They formed contact surfaces for the condensation of oxidized products resulting in the formation of secondary organic aerosols. These particles were produced from the nebulization of an ammonium sulfate solution through an atomizer. In order to realistically simulate an atmosphere representative of Beijing, soot particles were generated using a soot generator (miniCAST Series 5200, which produces combustion soot particles by using a well-defined flame that simulates the combustion in modern combustion engines), based on the combustion of propane. These particles were transferred into a smaller chamber (soot reservoir chamber) and were injected once a day into the CESAM chamber. Moreover, also once a day, mineral dust particles were injected, produced through a shaking process from a Gobi Desert sample, into the chamber (simulating a desert storm that impacted Beijing with a mineral dust plume).

Table 1 gives an overview of the pollutants defined as reference to qualify a "Beijing-like" atmosphere simulation. This shows how the atmospheric environment to which the mice were exposed is complex (tens of pollutants, both in aerosol and gaseous phases), and representative of the urban situation of Beijing in December 2013 (most of the reference pollutants are present in a relative abundance that is similar to the targeted range).

Table 1: Expected and observed ranges of December 2013 "Beijing-like" atmospheric pollutants. 


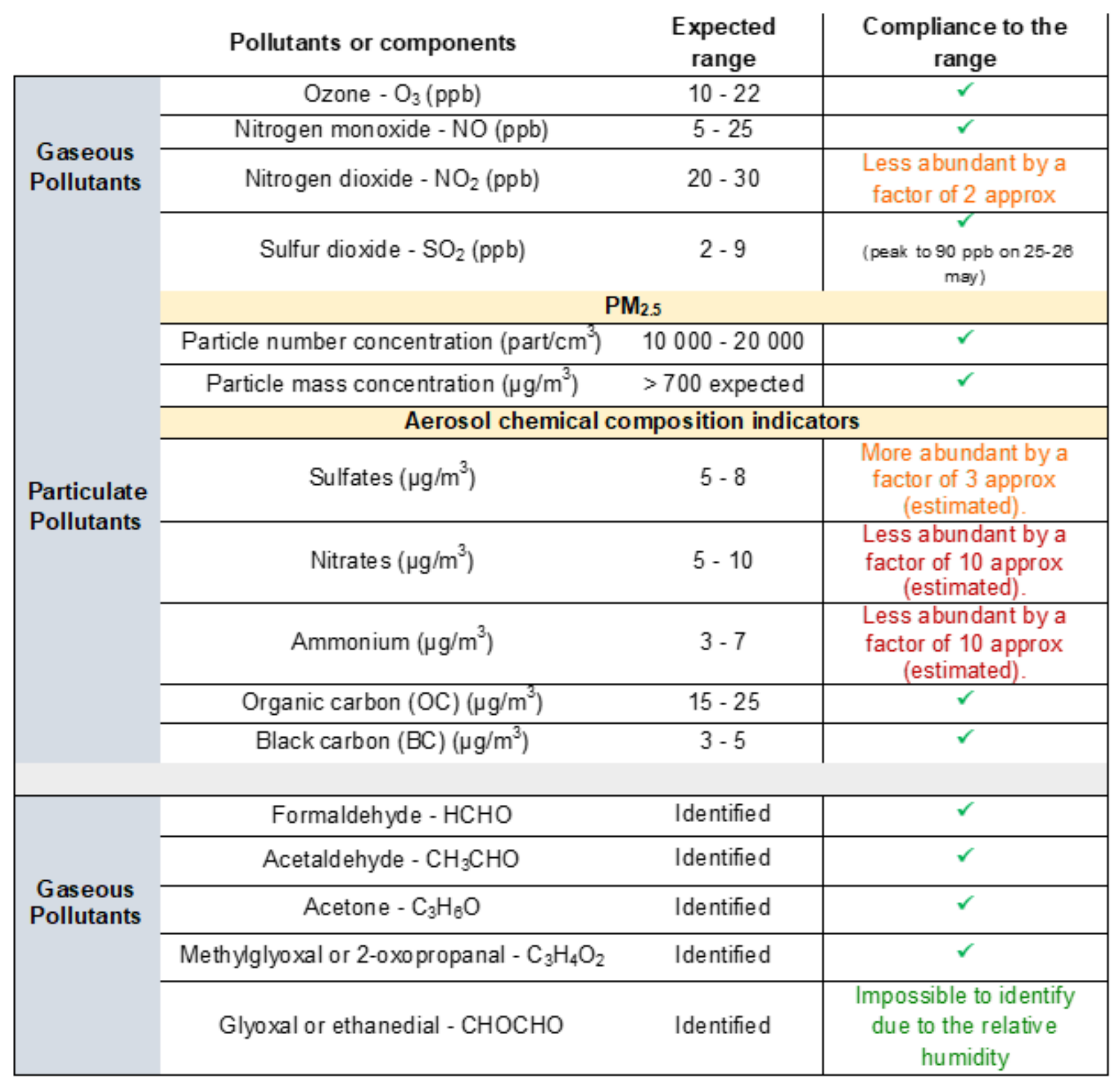

\section{Experimental design}

Primiparous pregnant C57/BL6J mice (Janvier labs) were exposed to simulated Beijing-like air $(n=8)$ or filtered Beijing-like air $(n=8)$ from GD10 to GD17. Water and food were given ad libitum. Then, the mice were housed under standard conventional conditions $\left(20^{\circ} \mathrm{C}\right.$, stable ambient humidity of $65 \%$, and imposed daylight cycle of $12 \mathrm{~h}$ of light and $12 \mathrm{~h}$ of darkness). The delivery occurred between GD19 and GD21. Male and female offspring was sacrificed on the 17th day of life. Intestinal tissues were sampled for histological and molecular biology analyses. The study was carried out in accordance with INSERM recommendations on the treatment of animals and was approved by the ethics committee of the University of Paris-Est Créteil Val de Marne (APAFiS N ${ }^{\circ}: 2017113013439718 ; N^{\circ}$ of Notice: 12/12 / 17-7).

\section{Histological analysis and image processing}


Proximal colon and ileum were fixed in 4\% paraformaldehyde overnight, processed, and embedded in paraffin wax by an automatic sample preparation system (LOGOS One, Milestone). Serial histological sections of $4 \mu \mathrm{m}$ thickness were cut, deparaffinized, rehydrated, and stained with MGG (Carlo Erba reagents, Val de Reuil, France, ref. E460583 and E453612). Images were acquired with a DM5500B microscope (Leica Microsystems, Nanterre, France) and intestinal layers were photographed at a magnification of 20x. Histomorphometric analyses were performed using Image $\mathrm{J}$ software. Submucosal cellularity and mucosal surface area in the colon were measured, as well as villus height and crypt depth in the ileum. At least 100 well-oriented mucosa, villi, and crypts were measured in at least 5 individual mice from each group. For vacuole morphologic analyses, an image processing algorithm developed by Lustig et al. was adapted [57]. This algorithm converts a microscope image from a red-green-blue image to a grayscale image: the vacuoles are colored white and all other components are blackened. Based on the overlay of the binary image and the grayscale-processed image, we selected clearly visible vacuoles and Image $\mathrm{J}$ allowed the measurement of 1 ) vacuole number; 2 ) vacuole area, automatically calculated in pixels and converted to $\mu \mathrm{m}^{2} ; 3$ ) vacuole circularity, a measurement of how closely the shape of the vacuoles approaches that of a circle (circularity can be valued between 0 and 1 inclusively, where 1 is the circularity value of an ideal circle); and 4) cell eccentricity, a measurement of how close the shape of the marked region approaches that of a line or a circle (eccentricity varies between 0 and 1 inclusively, where 0 is the eccentricity value of an ideal circle shape and 1 is the eccentricity value of a line segment).

\section{Immunohistochemical PCNA staining and quantification}

Serial histological sections of $4 \mu \mathrm{m}$ thickness were cut, deparaffinized, and rehydrated. For antigen unmasking, sections were placed in $10 \mathrm{mM}$ sodium citrate buffer $(\mathrm{pH} \mathrm{6.0)}$ ) and incubated in a heatinduced antigen retrieval chamber for $20 \mathrm{~min}$ at $121^{\circ} \mathrm{C}$. After washing, sections were blocked for $30 \mathrm{~min}$ with $5 \%$ bovine serum albumin in PBS. PCNA primary antibody (NB300-524, Novus Biologicals) was then incubated overnight at $4^{\circ} \mathrm{C}$ at a 1:1000 concentration. After washing, tissue sections were incubated for 1 $\mathrm{h}$ at room temperature with an Alexa fluor 647-conjugated anti-rabbit PCNA antibody (Thermo Fisher Scientific). Nuclear staining with Hoechst was performed before adding a fluorescent mounting medium. Microscopy was performed using a Leica DM5500 B microscope and data was processed with Leica LAS V3.8 software. Cells positive for PCNA were counted blindly by 2 investigators ( 5 crypts/slide, 1 slide/animal).

\section{Quantitative reverse transcription-PCR}

Small intestine, cecum, and colon tissue samples were homogenized with ceramic beads using Precellys lysing equipment (Bertin Technologies). Total RNA was extracted with the NucleoSpin RNA kit (MachereyNagel). Transcript levels of genes were quantified with the StepOne ${ }^{T M}$ Real-Time PCR System using a SYBR Green PCR master mix (Thermo Fisher Scientific). The primer sequences were designed using Primer Express 3 and are available upon request. Melting curve analyses were performed for each sample and gene. The relative expression of each target gene was normalized to the relative expression of the 
Polr2a housekeeping gene. Quantification of target gene expression was based on the comparative cycle threshold (Ct) value. Fold changes in target genes were analyzed by the $2^{-\Delta \Delta C t}$ method.

\section{Western blot}

Proteins were extracted from male ileum samples and homogenized in TRI Reagent ${ }^{\circledR}$ lysis buffer (SigmaAldrich) according to the manufacturer's instructions. Twenty micrograms of total protein lysate was separated by SDS-PAGE and electroblotted on nitrocellulose membranes using an Amersham Semi Dry Transfer Unit at $0.8 \mathrm{~mA} / \mathrm{cm}^{2}$ (Amersham Pharmacia Biotech). Membranes were blocked for $1 \mathrm{~h}$ in StartingBlock ${ }^{\text {TM }}$ T20 (TBS) with 5\% milk blocking buffer (Thermo Fisher Scientific) at room temperature. Then membranes were incubated overnight at $4^{\circ} \mathrm{C}$ with 1:500 of rabbit anti-ZO-1 (Thermo Fisher, 617300) and $1 \mathrm{~h}$ at room temperature with 1:10,000 of mouse anti-b-actin (Sigma, A1978). Immunoreactivity was visualized with SuperSignal ${ }^{\mathrm{TM}}$ West Pico Plus Chemiluminescent HRP Substrate (Thermo Fisher Scientific). Images were taken using the iBright FL1500 Imaging System (Thermo Fisher Scientific). For relative quantification, the volume intensity of the bands was obtained using iBright software.

\section{Bacterial DNA extraction and Illumina MiSeq sequencing}

Genomic DNA was extracted from the colon luminal content using a DNA stool kit (Macherey Nagel, France). The quantity and purity of DNA (expressed as the ratio of absorbance at $260 \mathrm{~nm}$ and $280 \mathrm{~nm}$ (A260/A280)) were assessed using a Nanodrop ${ }^{\circledR}$ spectrophotometer. The sequencing library was generated by amplifying the V3-V4 region of the bacterial 16S-rRNA gene using 16S rRNA amplicon generation for MiSeq with the primers Bact-0341 (CCTACGGGNGGCWGCAG) and Bact-0785 (GACTACHVGGGTATCTAATCC). Individual samples were barcoded, pooled to construct the sequencing library, and sequenced using an Illumina MiSeq (Illumina, San Diego, CA) to generate paired-end 2×300 bp reads.

\section{Analysis of sequencing data}

Bioinformatic analyses were performed using the QIIME2 pipeline (version 2020.2) [58]. The Divisive Amplicon Denoising Algorithm plug-in (DADA-2) in QIIME2 was used to filter, dereplicate, identify chimeric sequences, and merge PE reads to obtain a set of ASVs for each sample [59]. Then the representative sequences were picked for each ASV. The classify-sklearn plug-in in QIIME2, with the Silva database (version 132), was applied to assign a taxonomic annotation to each representative ASV sequence. In the next step, ASVs identified as eukaryotic contamination (3 ASVs; 12 reads) and external contamination, identified with the decontam package (3 ASVs; 3119 reads), were filtered out [60]. The diversity metrics (alpha and beta diversity) were obtained with the QIIME2 core-metrics-phylogenetic plug-in, with psampling depth parameter fixed to 13781 reads which corresponded to the total frequency that each sample should be rarefied to prior to computing diversity metrics. This sampling depth allowed retention of more than $61 \%$ of reads and only one sample was discarded. Tests for differential relative abundance were performed with corncob at the order, family, and genus levels [61]. 


\section{Statistics}

Results are expressed as mean \pm standard error of the mean. Except for metagenomic data, the statistical significance of differences between experimental groups was calculated using the Mann-Whitney nonparametric $U$ test (GraphPad Prism software, USA). Statistical significance was defined as $p<0.05$. For all experiments, ${ }^{*}=p<0.05, * \star=p<0.01, * \star \star=p<0.005$, and $=\star \star \star \star p<0.001$.

\section{Declarations}

\section{Ethics approval and consent to participate}

Not applicable

\section{Consent for publication}

Not applicable

\section{Availability of data and materials}

The datasets used and/or analyzed during the current study are available from the corresponding author on reasonable request.

\section{Competing interests}

The authors declare that they have no competing interests.

\section{Funding}

Mathilde Body-Malapel is the recipient of a grant from the Association François-Aupetit. Zhuyi Lu is the recipient of a grant from Fondation pour la Recherche Médicale. This work has received funding from the European Union's Horizon 2020 Research and Innovation Programme through the EUROCHAMP-2020 Infrastructure Activity under grant agreement $N^{\circ}$ 730997. We also thank CNRS/INSU, INSERM, Région Ile de France, Fondation du Crédit Agricole, Fondation du Souffle, and UPEC.

\section{Authors' contributions}

$E G, M D$, and $C W$ performed tissue analyses. $P C, M C, A B$, and $A G$ simulated Beijing-like air pollution, and ZL performed mice exposures. SG and DH were in charge of microbiota analysis. $L D, D L$, and $C V$ contributed to funding acquisition. SL conceptualized and supervised mice exposure. MB-M conceptualized and supervised tissue analyses. MB-M, SL, and PC wrote the original draft. All authors read and approved the final manuscript.

\section{Acknowledgements}


We deeply thank Elie Al Marj and Isabelle Coll for preliminary analysis regarding air quality data, and the Technical Department from LISA laboratory for continuous support regarding atmospheric simulation (Cécile Gaimoz, Edouard Pangui and Servanne Chevaillier especially). We thank Bernadette Leu for her broad-spectrum help. Editorial assistance, in the form of language editing and correction, was provided by XpertScientific Editing and Consulting Services.

\section{References}

1. Landrigan PJ, Fuller R, Acosta NJ, Adeyi O, Arnold R, Baldé AB, et al. The Lancet Commission on pollution and health. The lancet. Elsevier; 2018;391:462-512.

2. Lelieveld J, Pozzer A, Pöschl U, Fnais M, Haines A, Münzel T. Loss of life expectancy from air pollution compared to other risk factors: a worldwide perspective. Cardiovasc Res. 2020;

3. Al-Kindi SG, Brook RD, Biswal S, Rajagopalan S. Environmental determinants of cardiovascular disease: lessons learned from air pollution. Nat Rev Cardiol. Nature Publishing Group; 2020;17:65672.

4. Guan W-J, Zheng X-Y, Chung KF, Zhong N-S. Impact of air pollution on the burden of chronic respiratory diseases in China: time for urgent action. The Lancet. 2016;388:1939-51.

5. Huang S, Zhang X, Huang J, Lu X, Liu F, Gu D. Ambient air pollution and body weight status in adults: A systematic review and meta-analysis. Environ Pollut. 2020;265:114999.

6. Costa LG, Cole TB, Dao K, Chang Y-C, Coburn J, Garrick JM. Effects of air pollution on the nervous system and its possible role in neurodevelopmental and neurodegenerative disorders. Pharmacol Ther. 2020;210:107523.

7. Wu M-Y, Lo W-C, Chao C-T, Wu M-S, Chiang C-K. Association between air pollutants and development of chronic kidney disease: A systematic review and meta-analysis. Sci Total Environ. 2020;706:135522.

8. Orioli R, Solimini AG, Michelozzi P, Forastiere F, Davoli M, Cesaroni G. A cohort study on long-term exposure to air pollution and incidence of liver cirrhosis. Environ Epidemiol. 2020;4:e109.

9. Noorimotlagh Z, Azizi M, Pan H-F, Mami S, Mirzaee SA. Association between air pollution and Multiple Sclerosis: A systematic review. Environ Res. 2020;110386.

10. Alsaber A, Pan J, Al-Herz A, Alkandary DS, Al-Hurban A, Setiya P, et al. Influence of Ambient Air Pollution on Rheumatoid Arthritis Disease Activity Score Index. Int J Environ Res Public Health. Multidisciplinary Digital Publishing Institute; 2020;17:416.

11. Gilcrease GW, Padovan D, Heffler E, Peano C, Massaglia S, Roccatello D, et al. Is air pollution affecting the disease activity in patients with systemic lupus erythematosus? State of the art and a systematic literature review. Eur J Rheumatol. 2020;7:31-4.

12. Blaskievicz PH, Silva AMC, Fernandes V, Junior OBP, Shimoya-Bittencourt W, Ferreira SMB, et al. Atmospheric Pollution Exposure Increases Disease Activity of Systemic Lupus Erythematosus. Int $J$ Environ Res Public Health. Multidisciplinary Digital Publishing Institute; 2020;17:1984. 
13. Vignal C, Guilloteau E, Gower-Rousseau C, Body-Malapel M. Review article: Epidemiological and animal evidence for the role of air pollution in intestinal diseases. Sci Total Environ. 2021;757:143718.

14. Feng J, Cavallero S, Hsiai T, Li R. Impact of air pollution on intestinal redox lipidome and microbiome. Free Radic Biol Med. 2020;

15. Kim Juyong Brian, Prunicki Mary, Haddad Francois, Dant Christopher, Sampath Vanitha, Patel Rushali, et al. Cumulative Lifetime Burden of Cardiovascular Disease From Early Exposure to Air Pollution. J Am Heart Assoc. American Heart Association; 2020;9:e014944.

16. Rosa MJ, Hair GM, Just AC, Kloog I, Svensson K, Pizano-Zárate ML, et al. Identifying critical windows of prenatal particulate matter (PM2.5) exposure and early childhood blood pressure. Environ Res. 2020;182:109073.

17. Deng Q, Lu C, Li Y, Sundell J, Dan Norbäck. Exposure to outdoor air pollution during trimesters of pregnancy and childhood asthma, allergic rhinitis, and eczema. Environ Res. 2016;150:119-27.

18. Lu C, Norbäck D, Li Y, Deng Q. Early-life exposure to air pollution and childhood allergic diseases: an update on the link and its implications. Expert Rev Clin Immunol. Taylor \& Francis; 2020;16:813-27.

19. Elten M, Benchimol El, Fell DB, Kuenzig ME, Smith $G$, Chen $H$, et al. Ambient air pollution and the risk of pediatric-onset inflammatory bowel disease: A population-based cohort study. Environ Int. 2020;138:105676.

20. Liu W, Zhou Y, Yong li, Qin Y, Yu L, Li R, et al. Effects of PM2.5 exposure during gestation on maternal gut microbiota and pregnancy outcomes. Chemosphere. 2020;247:125879.

21. Beaumont M, Paës $C$, Mussard E, Knudsen C, Cauquil L, Aymard P, et al. Gut microbiota derived metabolites contribute to intestinal barrier maturation at the suckling-to-weaning transition. Gut Microbes. Taylor \& Francis; 2020;11:1268-86.

22. Navis M, Martins Garcia T, Renes IB, Vermeulen JL, Meisner S, Wildenberg ME, et al. Mouse fetal intestinal organoids: new model to study epithelial maturation from suckling to weaning. EMBO Rep. John Wiley \& Sons, Ltd; 2019;20:e46221.

23. Sureda EA, Weström B, Pierzynowski SG, Prykhodko O. Maturation of the Intestinal Epithelial Barrier in Neonatal Rats Coincides with Decreased FcRn Expression, Replacement of Vacuolated Enterocytes and Changed Blimp-1 Expression. PLOS ONE. Public Library of Science; 2016;11:e0164775.

24. Garcia TM, Navis M, Wildenberg ME, van Elburg RM, Muncan V. Recapitulating Suckling-to-Weaning Transition In Vitro using Fetal Intestinal Organoids. JoVE J Vis Exp. 2019;e60470.

25. Muncan V, Heijmans J, Krasinski SD, Büller NV, Wildenberg ME, Meisner S, et al. Blimp1 regulates the transition of neonatal to adult intestinal epithelium. Nat Commun. 2011;2:452.

26. Harper J, Mould A, Andrews RM, Bikoff EK, Robertson EJ. The transcriptional repressor Blimp1/Prdm1 regulates postnatal reprogramming of intestinal enterocytes. Proc Natl Acad Sci U S A. 2011;108:10585-90. 
27. Beumer J, Artegiani B, Post Y, Reimann F, Gribble F, Nguyen TN, et al. Enteroendocrine cells switch hormone expression along the crypt-to-villus BMP signalling gradient. Nat Cell Biol. Nature Publishing Group; 2018;20:909-16.

28. Coll P, Cazaunau M, Boczkowski J, Zysman M, Doussin J-F, Gratien A, et al. POLLURISK: AN INNOVATIVE EXPERIMENTAL PLATFORM TO INVESTIGATE HEALTH IMPACTS OF AIR QUALITY. Naples, Italy; 2018 [cited 2021 Feb 23]. p. 557-65. Available from: http://library.witpress.com/viewpaper.asp?pcode=AIR18-052-1

29. Noah TK, Donahue B, Shroyer NF. Intestinal development and differentiation. Exp Cell Res. 2011;317:2702-10.

30. Sumigray KD, Terwilliger M, Lechler T. Morphogenesis and Compartmentalization of the Intestinal Crypt. Dev Cell. 2018;45:183-197.e5.

31. Naik S, Larsen SB, Gomez NC, Alaverdyan K, Sendoel A, Yuan S, et al. Inflammatory memory sensitizes skin epithelial stem cells to tissue damage. Nature. 2017;550:475-80.

32. Ge Y, Gong M, Colliou N, Zadeh M, Li J, Jones DP, et al. Neonatal intestinal immune regulation by the commensal bacterium, P. UF1. Mucosal Immunol. Nature Publishing Group; 2019;12:434-44.

33. El Aidy S, Van Baarlen P, Derrien M, Lindenbergh-Kortleve DJ, Hooiveld G, Levenez F, et al. Temporal and spatial interplay of microbiota and intestinal mucosa drive establishment of immune homeostasis in conventionalized mice. Mucosal Immunol. Nature Publishing Group; 2012;5:567-79.

34. Köhler A, Delbauve S, Smout J, Torres D, Flamand V. Very early-life exposure to microbiota-induced TNF drives the maturation of neonatal pre-cDC1. Gut. BMJ Publishing Group; 2021;70:511-21.

35. Razzaghian HR, Sharafian Z, Sharma AA, Boyce GK, Lee K, Da Silva R, et al. Neonatal T Helper 17 Responses Are Skewed Towards an Immunoregulatory Interleukin-22 Phenotype. Front Immunol [Internet]. 2021 [cited 2021 Jun 17];12. Available from:

https://www.ncbi.nlm.nih.gov/pmc/articles/PMC8126652/

36. Mirpuri J. The emerging role of group 3 innate lymphoid cells in the neonate: interaction with the maternal and neonatal microbiome. Oxf Open Immunol [Internet]. 2021 [cited 2021 Jun 17];2. Available from: https://doi.org/10.1093/oxfimm/iqab009

37. Chen Y-S, Chen I-B, Pham G, Shao T-Y, Bangar H, Way SS, et al. IL-17-producing y $\delta$ T cells protect against Clostridium difficile infection. J Clin Invest. American Society for Clinical Investigation; 2020;130:2377-90.

38. Weström B, Arévalo Sureda E, Pierzynowska K, Pierzynowski SG, Pérez-Cano F-J. The Immature Gut Barrier and Its Importance in Establishing Immunity in Newborn Mammals. Front Immunol [Internet]. Frontiers; 2020 [cited 2021 Jun 17];11. Available from: https://www.frontiersin.org/articles/10.3389/fimmu.2020.01153/full

39. Akdis CA. Does the epithelial barrier hypothesis explain the increase in allergy, autoimmunity and other chronic conditions? Nat Rev Immunol. 2021;1-13.

40. Sarkar A, Yoo JY, Valeria Ozorio Dutra S, Morgan KH, Groer M. The Association between Early-Life Gut Microbiota and Long-Term Health and Diseases. J Clin Med. Multidisciplinary Digital Publishing 
Institute; 2021;10:459.

41. Stojanov S, Berlec A, Štrukelj B. The Influence of Probiotics on the Firmicutes/Bacteroidetes Ratio in the Treatment of Obesity and Inflammatory Bowel disease. Microorganisms. Multidisciplinary Digital Publishing Institute; 2020;8:1715.

42. Low A, Soh M, Miyake S, Seedorf H. Host-age prediction from fecal microbiome composition in laboratory mice. bioRxiv. Cold Spring Harbor Laboratory; 2020;2020.12.04.412734.

43. Dou X, Gao N, Yan D, Shan A. Sodium Butyrate Alleviates Mouse Colitis by Regulating Gut Microbiota Dysbiosis. Animals. Multidisciplinary Digital Publishing Institute; 2020;10:1154.

44. Chen Q, Wang Y, Jiao F, Shi C, Pei M, Wang L, et al. Betaine inhibits Toll-like receptor 4 responses and restores intestinal microbiota in acute liver failure mice. Sci Rep. 2020;10:21850.

45. Chiodini RJ, Dowd SE, Chamberlin WM, Galandiuk S, Davis B, Glassing A. Microbial Population Differentials between Mucosal and Submucosal Intestinal Tissues in Advanced Crohn's Disease of the lleum. PLOS ONE. Public Library of Science; 2015;10:e0134382.

46. Oezguen N, Yalcinkaya N, Kücükali Cl, Dahdouli M, Hollister EB, Luna RA, et al. Microbiota stratification identifies disease-specific alterations in neuro-Behcet's disease and multiple sclerosis. Clin Exp Rheumatol. 2019;37:S58-66.

47. Munyaka PM, Rabbi MF, Khafipour E, Ghia J-E. Acute dextran sulfate sodium (DSS)-induced colitis promotes gut microbial dysbiosis in mice. J Basic Microbiol. 2016;56:986-98.

48. Kuhn KA, Schulz HM, Regner EH, Severs EL, Hendrickson JD, Mehta G, et al. Bacteroidales recruit IL-6producing intraepithelial lymphocytes in the colon to promote barrier integrity. Mucosal Immunol. 2018;11:357-68.

49. Coyne MJ, Béchon N, Matano LM, McEneany VL, Chatzidaki-Livanis M, Comstock LE. A family of anti-Bacteroidales peptide toxins wide-spread in the human gut microbiota. Nat Commun. 2019;10:3460.

50. Zitomersky NL, Atkinson BJ, Franklin SW, Mitchell PD, Snapper SB, Comstock LE, et al. Characterization of Adherent Bacteroidales from Intestinal Biopsies of Children and Young Adults with Inflammatory Bowel Disease. PLOS ONE. Public Library of Science; 2013;8:e63686.

51. Bolton JL, Smith SH, Huff NC, Gilmour MI, Foster WM, Auten RL, et al. Prenatal air pollution exposure induces neuroinflammation and predisposes offspring to weight gain in adulthood in a sex-specific manner. FASEB J. 2012;26:4743-54.

52. Thaver S, Foa L, Richards SM, Lyons AB, Zosky GR. In utero exposure to diesel exhaust particles, but not silica, alters post-natal immune development and function. Chemosphere. 2021;268:129314.

53. Clougherty Jane E. A Growing Role for Gender Analysis in Air Pollution Epidemiology. Environ Health Perspect. Environmental Health Perspectives; 2010;118:167-76.

54. Shukla A, Bunkar N, Kumar R, Bhargava A, Tiwari R, Chaudhury K, et al. Air pollution associated epigenetic modifications: Transgenerational inheritance and underlying molecular mechanisms. Sci Total Environ. 2019;656:760-77. 
55. Tsamou M, Vrijens K, Madhloum N, Lefebvre W, Vanpoucke C, Nawrot TS. Air pollution-induced placental epigenetic alterations in early life: a candidate miRNA approach. Epigenetics. 2018;13:135-46.

56. Vrijens K, Trippas A-J, Lefebvre W, Vanpoucke C, Penders J, Janssen BG, et al. Association of Prenatal Exposure to Ambient Air Pollution With Circulating Histone Levels in Maternal Cord Blood. JAMA Netw Open. 2020;3:e205156-e205156.

57. Lustig M, Feng Q, Payan Y, Gefen A, Benayahu D. Noninvasive continuous monitoring of adipocyte differentiation: From macro to micro scales. Microsc Microanal. Cambridge University Press; 2019;25:119-28.

58. Bolyen E, Rideout JR, Dillon MR, Bokulich NA, Abnet CC, Al-Ghalith GA, et al. Reproducible, interactive, scalable and extensible microbiome data science using QIIME 2. Nat Biotechnol. 2019;37:852-7.

59. Callahan BJ, McMurdie PJ, Rosen MJ, Han AW, Johnson AJA, Holmes SP. DADA2: High-resolution sample inference from Illumina amplicon data. Nat Methods. 2016;13:581-3.

60. Davis NM, Proctor DM, Holmes SP, Relman DA, Callahan BJ. Simple statistical identification and removal of contaminant sequences in marker-gene and metagenomics data. Microbiome. 2018;6:226.

61. Martin BD, Witten D, Willis AD. MODELING MICROBIAL ABUNDANCES AND DYSBIOSIS WITH BETABINOMIAL REGRESSION. Ann Appl Stat. 2020;14:94-115.

\section{Figures}




\section{Proximal colon}
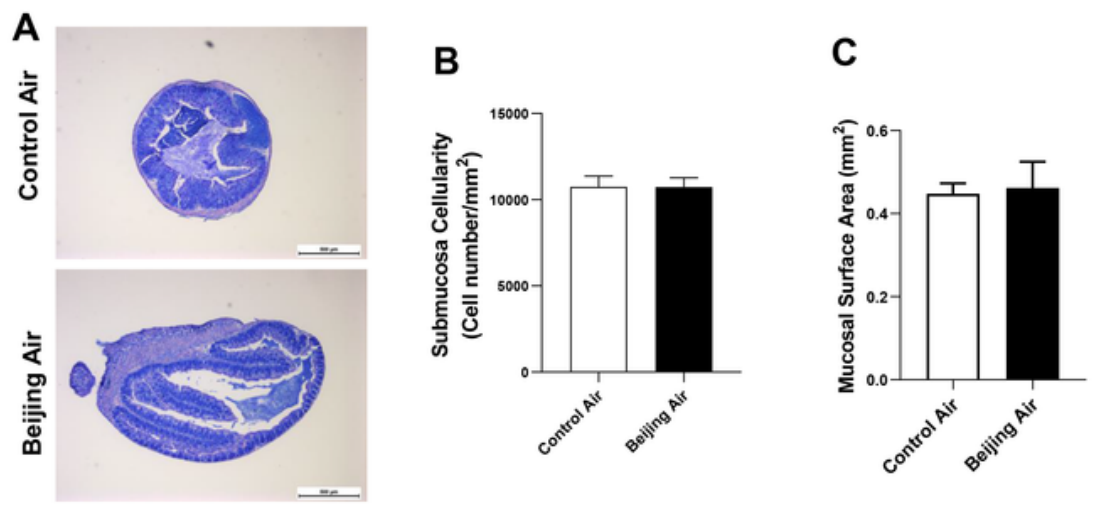

lleum

D
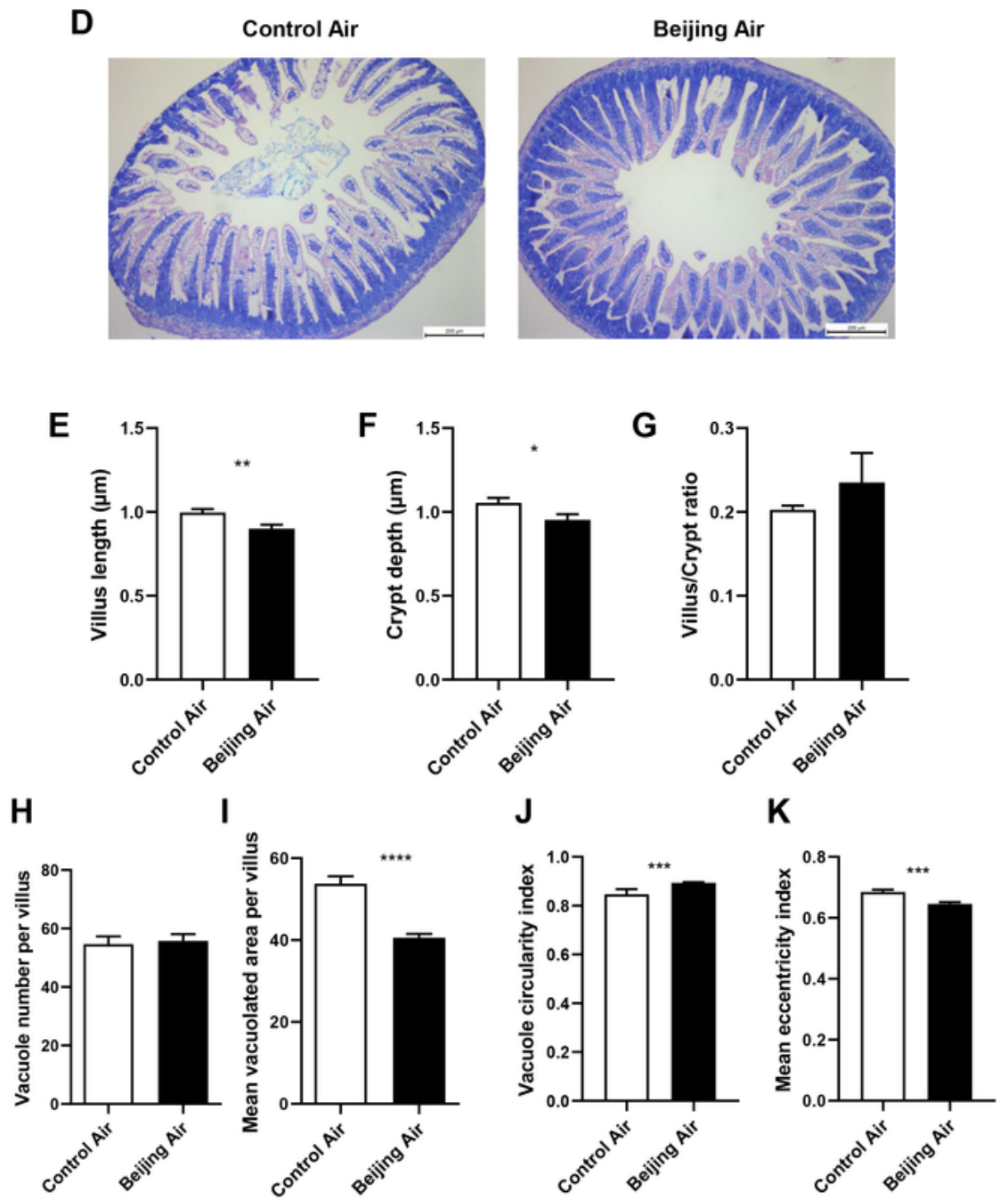

\section{Figure 1}

Histomorphological analysis in male mice. A Representative pictures of MGG-stained proximal colon from control air- and Beijing-like air-exposed mice, scale bar $500 \mu \mathrm{m}$ ( $\mathrm{n}=10 / \mathrm{group})$. B Submucosa cellularity. C Mucosal surface area. D Representative pictures of MGG-stained ileum from control air- and Beijing-like air-exposed mice, scale bar $200 \mu \mathrm{m}$ (n=10/group). E Villus length. F Crypt depth. G Villus/crypt ratio. H Vacuole number per villus. I Mean vacuolated area per villus. J Vacuole circularity index. K Mean 
eccentricity index. ${ }^{*} p<0.05,{ }^{*}$ p $<0.01,{ }^{* \star *} p<0.005,{ }^{* \star * *} p<0.001$ as determined by the Mann-Whitney U test.

\section{Proximal colon}
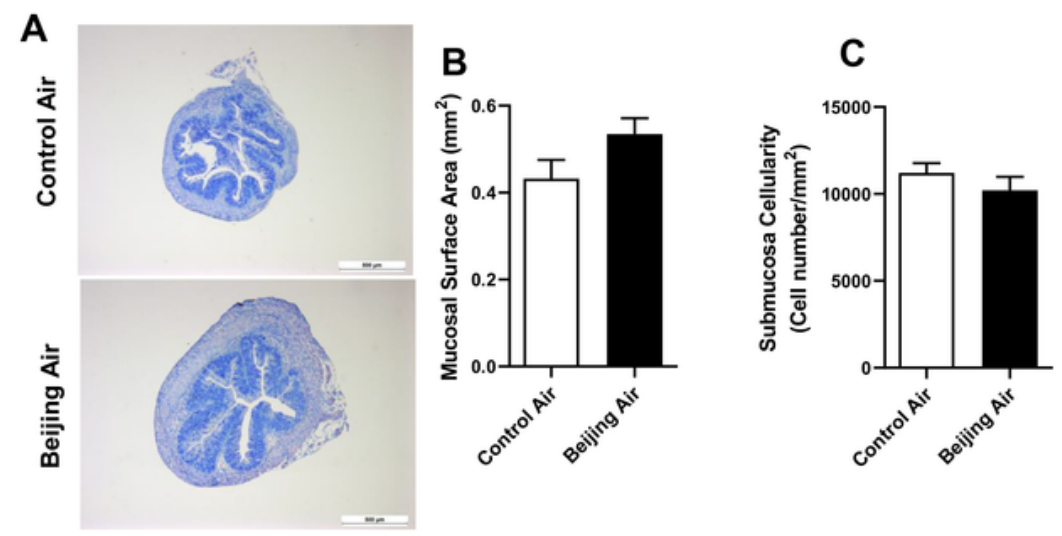

Ileum

D
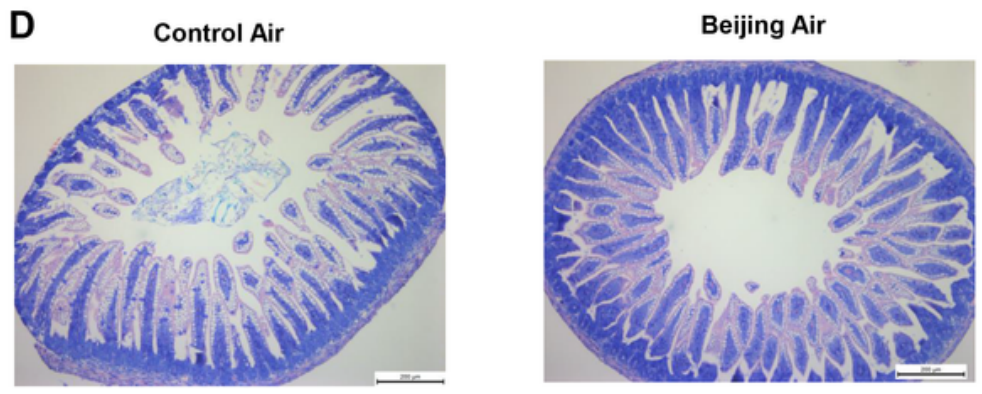

E

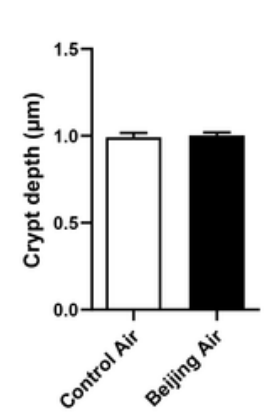

F

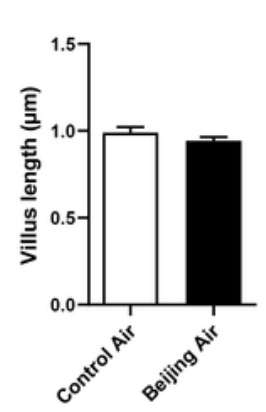

H

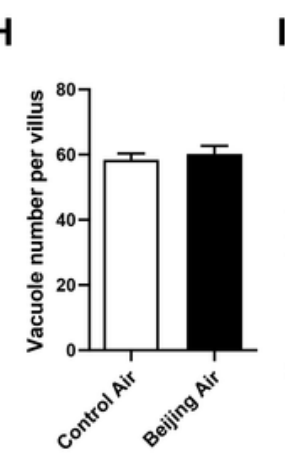

I

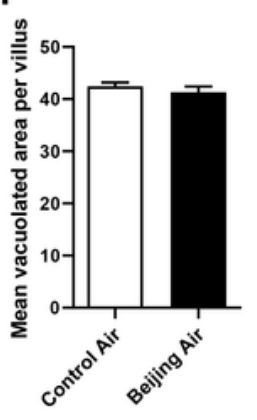

$\mathbf{J}$

G

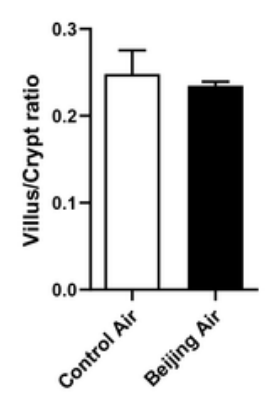

$\mathrm{K}$
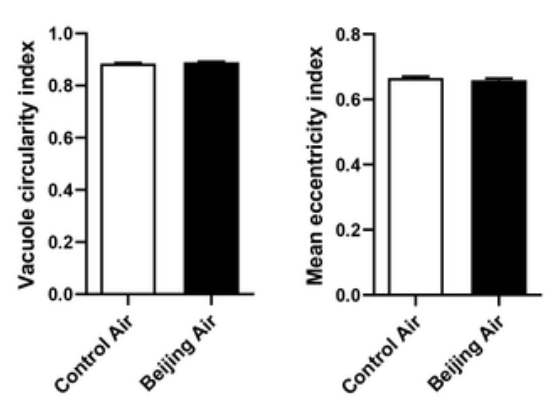

Figure 2

Histomorphological analysis in female mice. A Representative pictures of MGG-stained proximal colon from control air- and Beijing-like air-exposed mice, scale bar $500 \mu \mathrm{m}$ ( $\mathrm{n}=10 /$ group). B Submucosa cellularity. C Mucosal surface area. D Representative pictures of MGG-stained ileum control air- and 
Beijing-like air-exposed mice, scale bar $200 \mu \mathrm{m}$ (n=10/group). E Villus length. F Crypt depth. G Villus/crypt ratio. $\mathrm{H}$ Vacuole number per villus. I Mean vacuolated area per villus. J Vacuole circularity index. K Mean eccentricity index.

\section{Proximal colon}

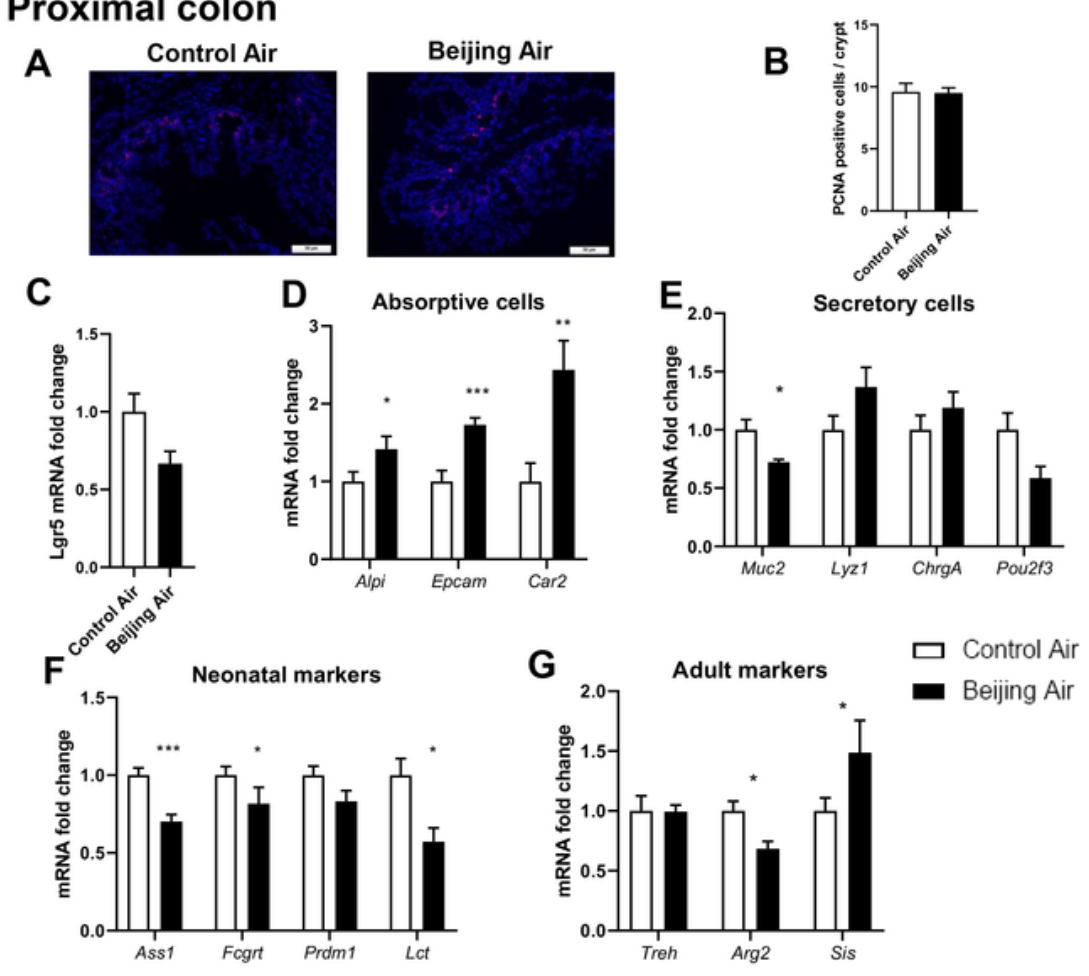

\section{lleum}

H

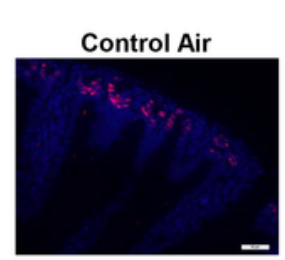

$\mathbf{J}$

K

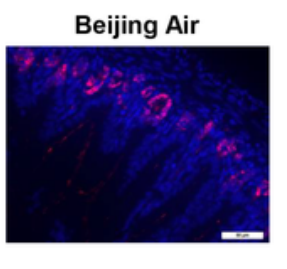

L
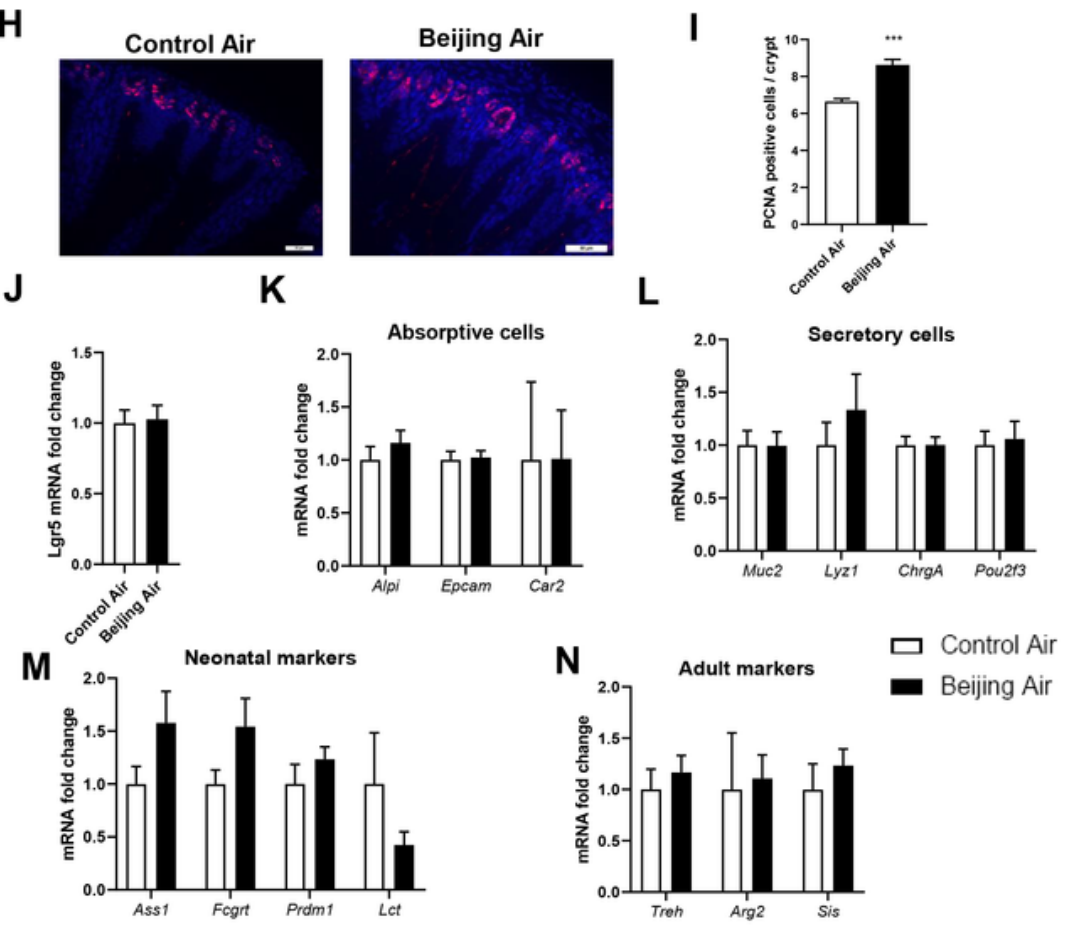

Figure 3

Epithelium proliferation, differentiation, and maturation in male mice. A Representative pictures of PCNAstained proximal colon from control air- and Beijing-like air-exposed mice, scale bar $50 \mu \mathrm{m}$ ( $\mathrm{n}=10$ /group). 
B Number of PCNA positive cells/crypt. C Lgr5 transcript levels. D Alpi, Epcam, and Car2 transcript levels. E Muc2, Lyz1, ChrgA, and Pou2f3 transcript levels. F Ass1, Fcgrt, Prdm1, and Lct transcript levels. G Treh, Arg2, and Sis transcript levels. H Representative pictures of PCNA-stained ileum from control air- and Beijing-like air-exposed mice, scale bar $50 \mu \mathrm{m}$ ( $\mathrm{n}=10$ /group). I Number of PCNA-positive cells/crypt. J Lgr5 transcript levels. K Alpi, Epcam, and Car2 transcript levels. L Muc2, Lyz1, ChrgA, and Pou2f3 transcript levels. M Ass1, Fcgrt, Prdm1, and Lct transcript levels. N Treh, Arg2, and Sis transcript levels. * $p<0.05$, $* \star p<0.01$, $* \star \star ~ p<0.005$ as determined by the Mann-Whitney $U$ test.

\section{Proximal colon}

A

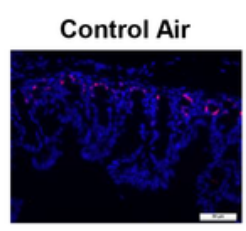

C
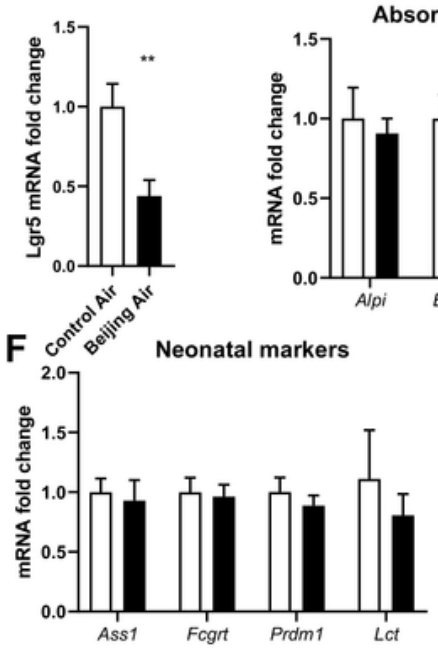

Beijing Air

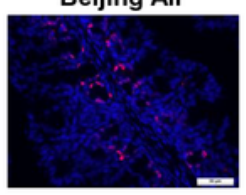

D

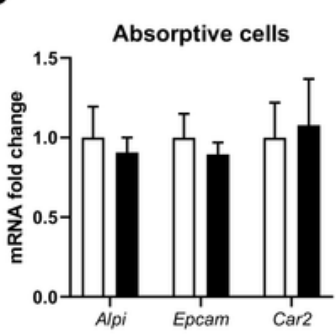

E
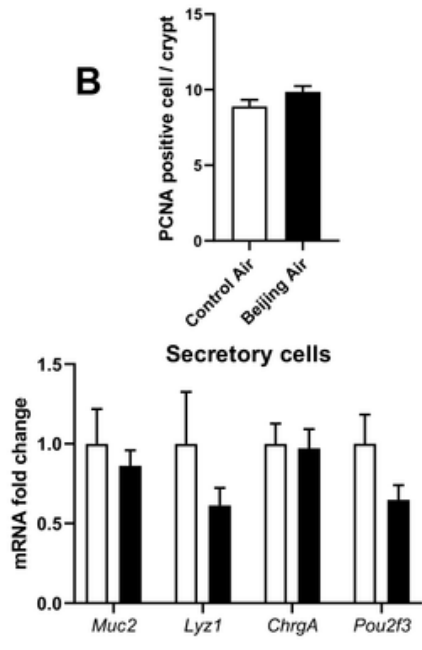

G

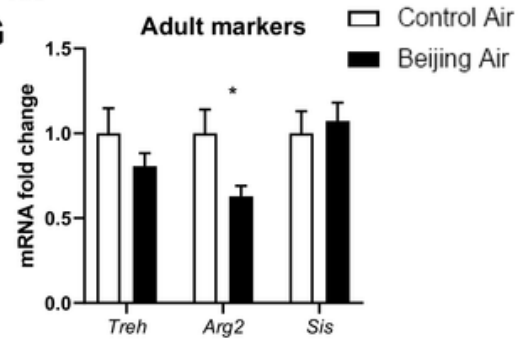

\section{Ileum}

H

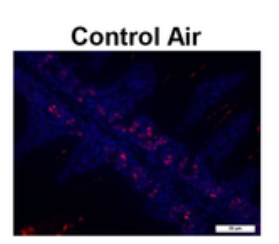

J

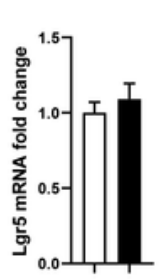

K

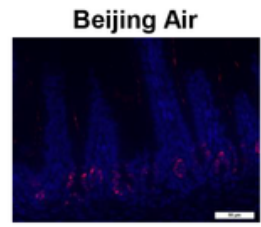

Absorptive cells

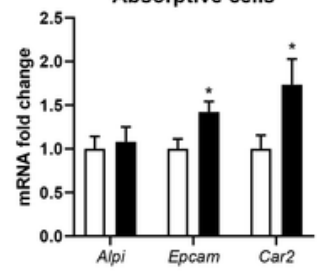

L

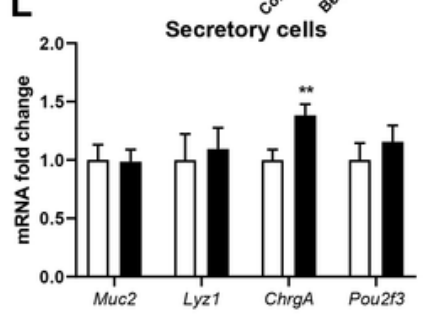

M

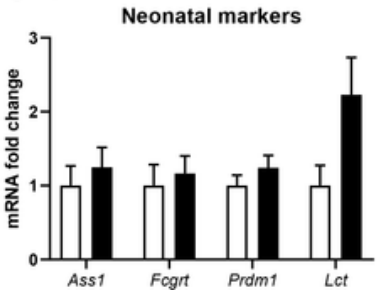

$\mathbf{N}$
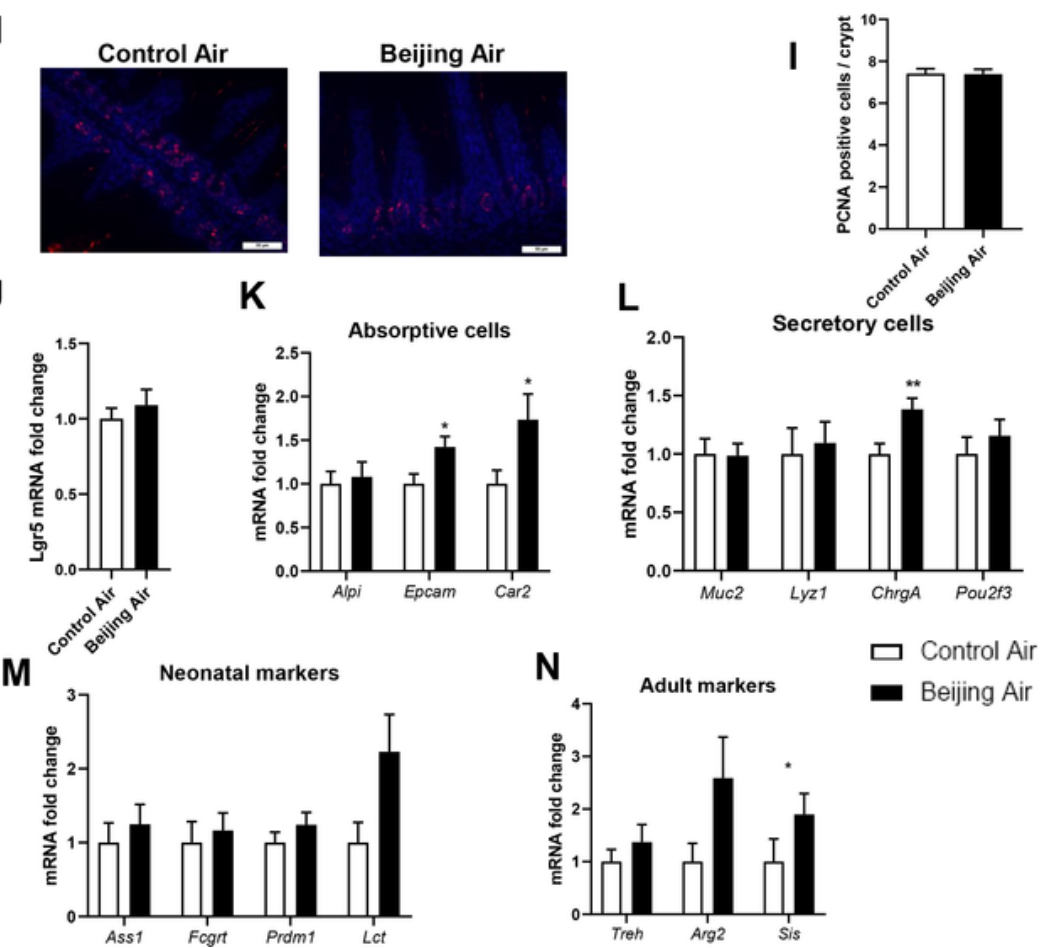


\section{Figure 4}

Epithelium proliferation, differentiation, and maturation in female mice. A Representative pictures of PCNA-stained proximal colon from control air- and Beijing-like air-exposed mice, scale bar $50 \mu \mathrm{m}$ ( $n=10 /$ group). B Number of PCNA-positive cells/crypt. C Lgr5 transcript levels. D Alpi, Epcam, and Car2 transcript levels. E Muc2, Lyz1, ChrgA, and Pou2f3 transcript levels. F Ass1, Fcgrt, Prdm1, and Lct transcript levels. G Treh, Arg2, and Sis transcript levels. H Representative pictures of PCNA-stained ileum from control air- and Beijing-like air-exposed mice, scale bar $50 \mu \mathrm{m}$ ( $n=10 /$ group). I Number of PCNApositive cells/crypt. J Lgr5 transcript levels. K Alpi, Epcam, and Car2 transcript levels. L Muc2, Lyz1, ChrgA, and Pou2f3 transcript levels. M Ass1, Fcgrt, Prdm1, and Lct transcript levels. N Treh, Arg2, and Sis transcript levels. ${ }^{*} p<0.05,{ }^{*} p<0.01$ as determined by the Mann-Whitney $U$ test. 
A Male Proximal Colon

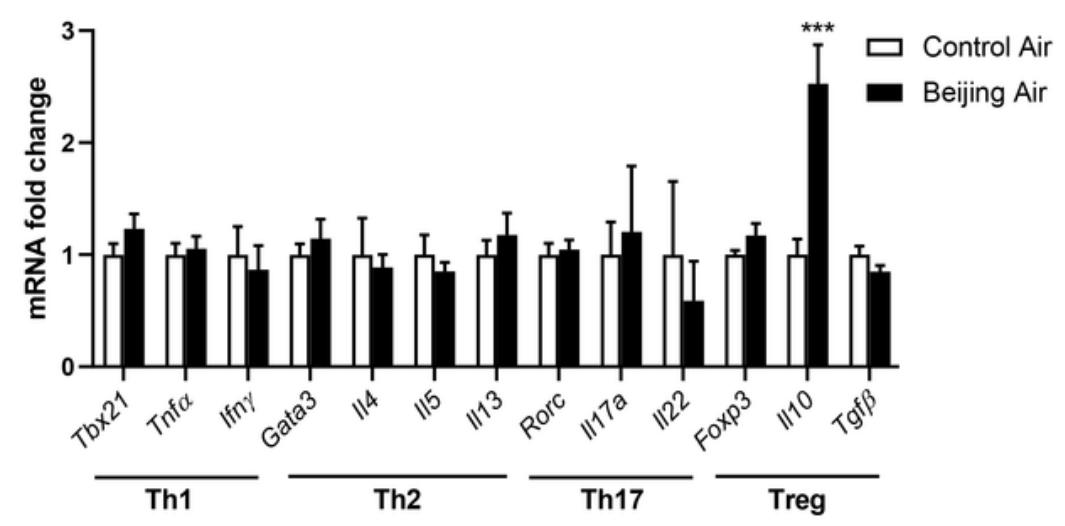

B

Male Cecum

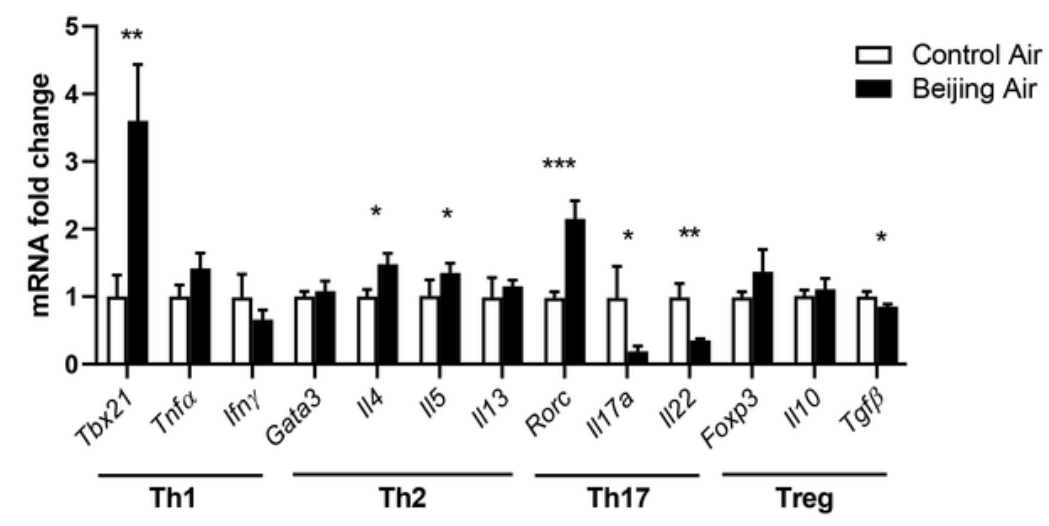

C

Male Ileum

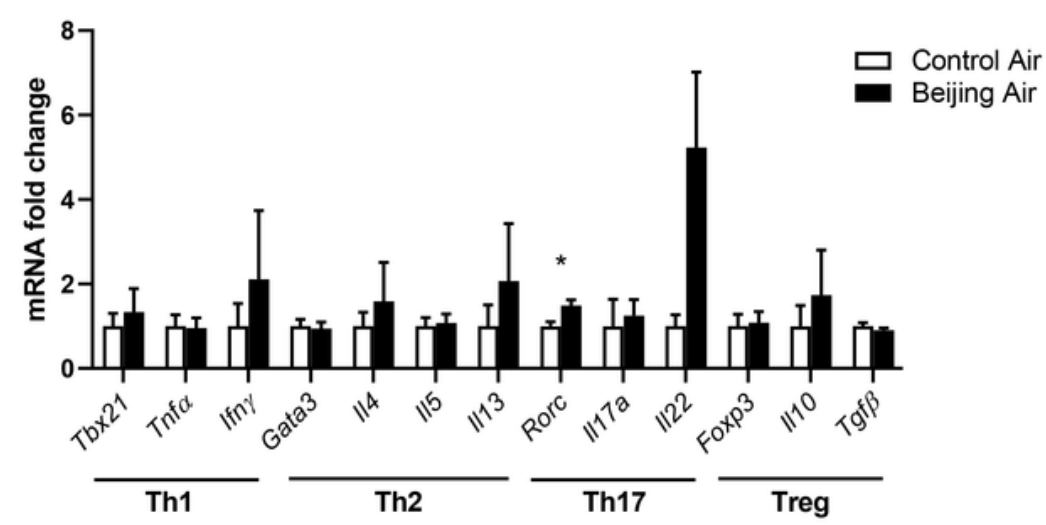

Figure 5

Inflammatory pattern in male mice. Transcript levels of Th1 (Tbx21, Tnfa, Ifny), Th2 (Gata3, II4, II5, II13), Th17 (Rorc, II17a, II22), and Treg (Foxp3, Il10, Tgf $\beta$ ) genes in proximal colon (A), cecum (B), and ileum (C) from control air- and Beijing-like air-exposed male mice ( $n=10 /$ group). ${ }^{\star} p<0.05,{ }^{\star \star} p<0.01,{ }^{\star \star \star} p<0.005$ as determined by the Mann-Whitney $U$ test. 
A

Female Proximal Colon

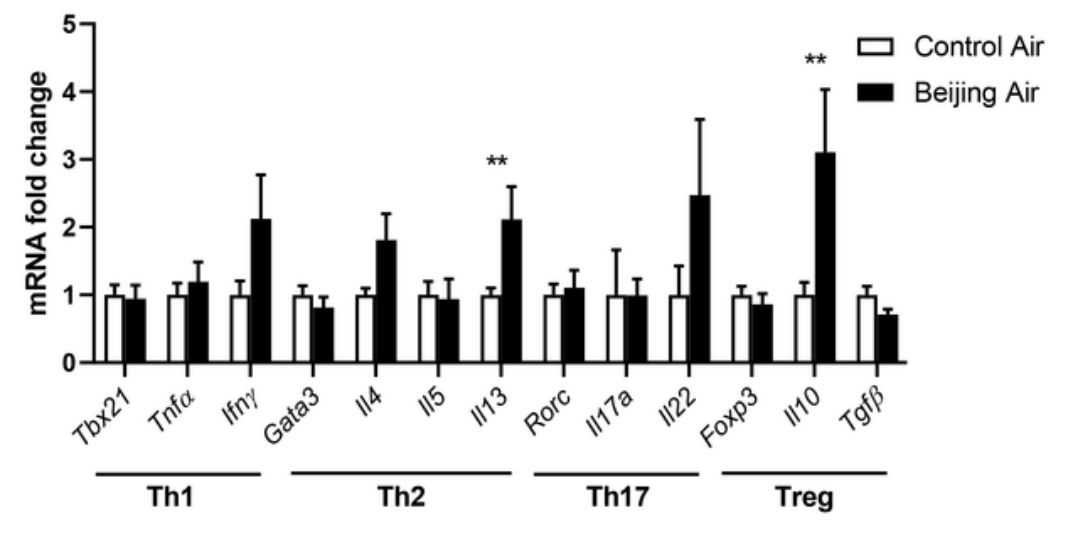

B

Female Cecum

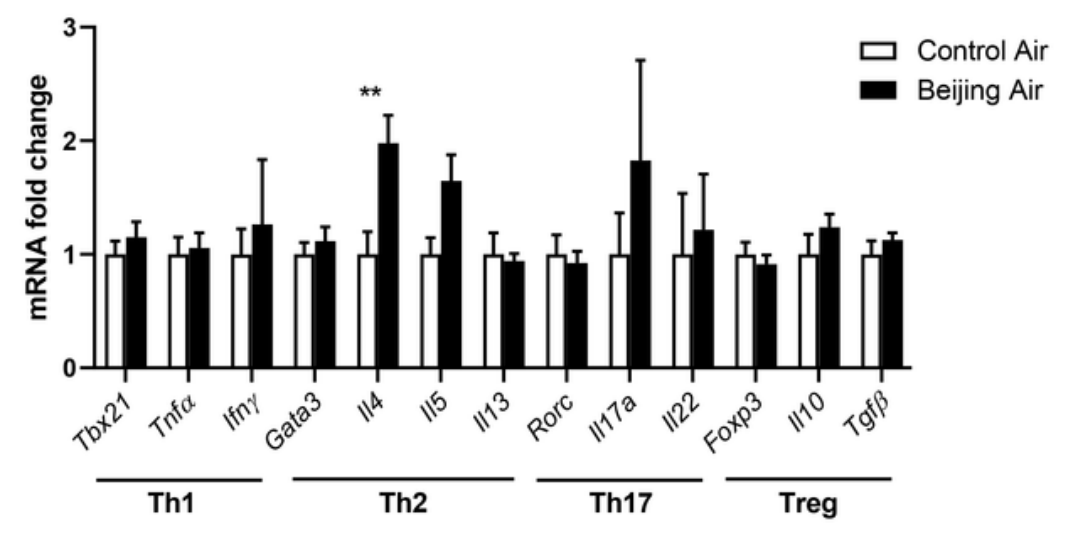

C

Female Ileum

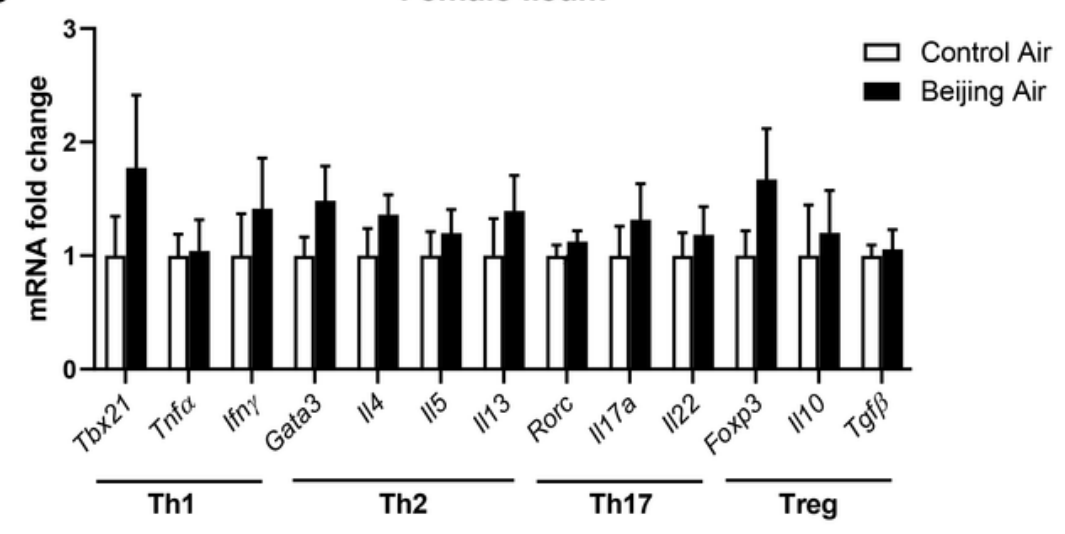

Figure 6

Inflammatory pattern in female mice. Transcript levels of Th1 (Tbx21, Tnfa, Ifny), Th2 (Gata3, II4, II5, II13), Th17 (Rorc, II17a, II22), and Treg (Foxp3, II10, Tgf $\beta$ ) genes in proximal colon (A), cecum (B), and ileum (C) from control air- and Beijing-like air-exposed male mice ( $n=10 /$ group). ${ }^{* \star} p<0.01$ as determined by the Mann-Whitney U test. 
A
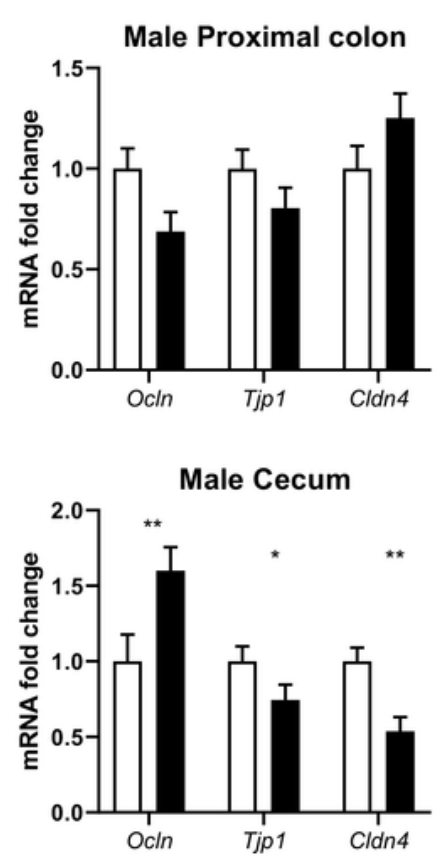

Male Ileum

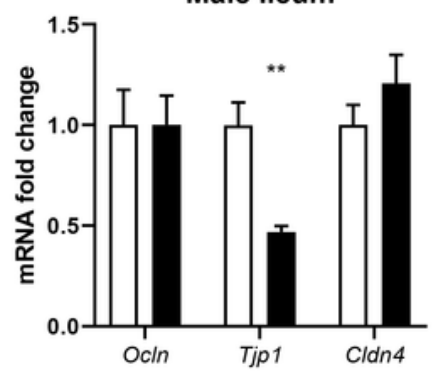

C

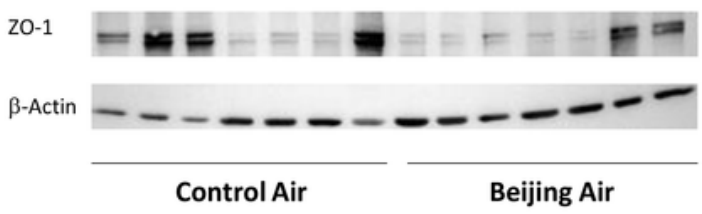

B
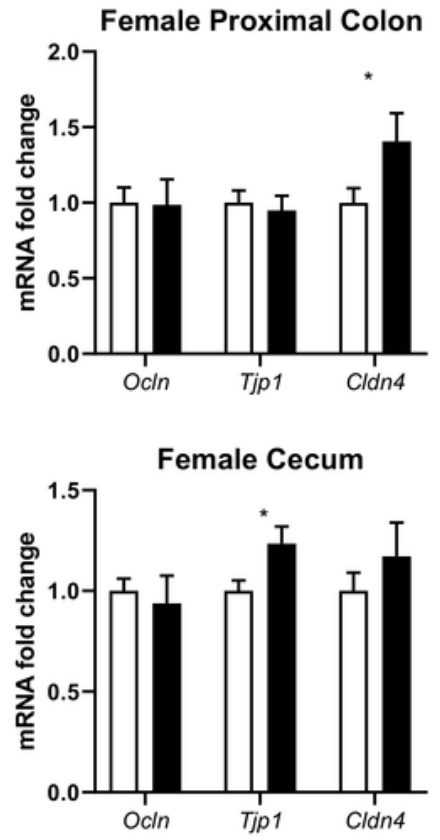

Female lleum

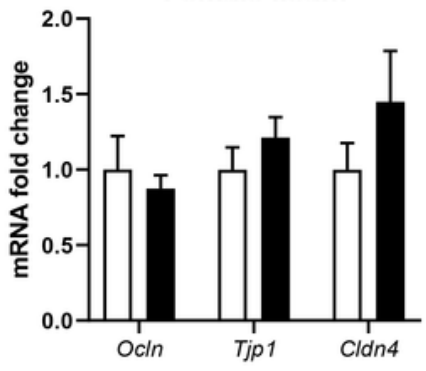

D

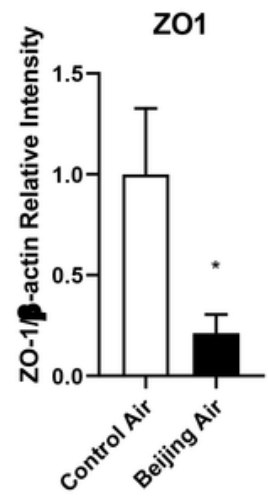

Figure 7

Tight junction expression in male and female mice. A-B Transcript levels of Ocln, Tjp1 and Cldn4 in proximal colon, cecum, and ileum of male (A) and female (B) mice exposed to control air or Beijing-like air ( $n=10$ /group). C ZO-1 and $\beta$-actin western blot analysis of male ileum. D Densitometric analysis of ZO-1 western blots. ${ }^{\star} p<0.05,{ }^{*} p<0.01$ as determined by the Mann-Whitney U test. 
A

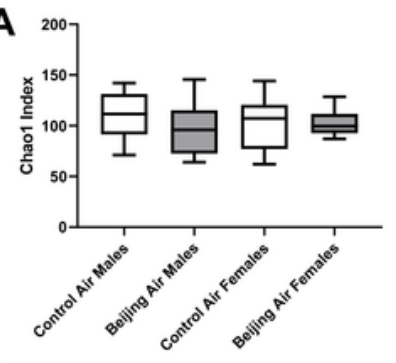

B

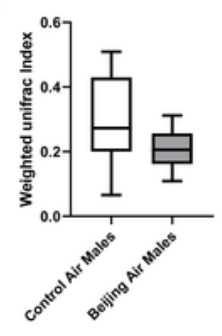

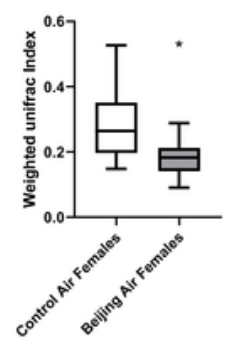

C

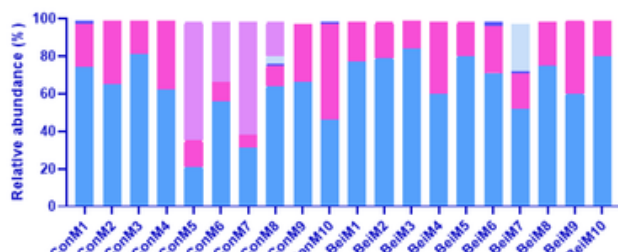

$$
\begin{aligned}
& \text { Unassigned } \\
& \text { Patescibacteria } \\
& \text { Tenericutes } \\
& \text { Verrucomicrobia } \\
& \text { Actinobacteria } \\
& \text { Proteobacteria } \\
& \text { Firmicutes } \\
& \text { Bacteroidetes }
\end{aligned}
$$

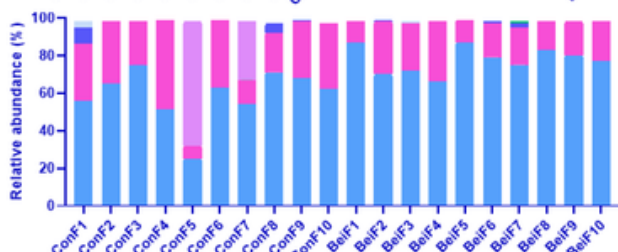

D

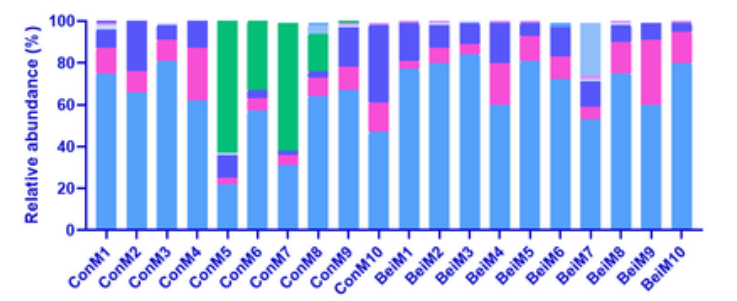

E... Unassigned

n- Mollicutes RF39

- Saccharimonadales

EN Pseudomonadales

-.... Anaeroplasmatales

- Enterobacteriales

- Bacillales

- Desulfovibrionales

- Coriobacteriales

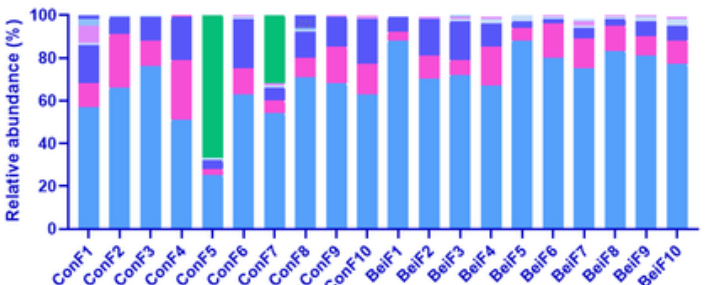

nicrococcales

- Bifidobacteriales

- Verrucomicrobiales

- Betaproteobacteriales

Erysipelotrichales

- Lactobacillales

- Clostridiales

- Bacteroidales

E

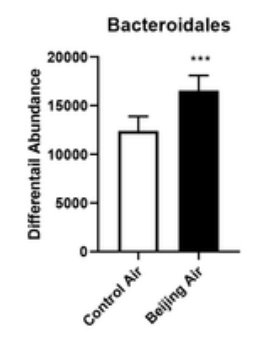

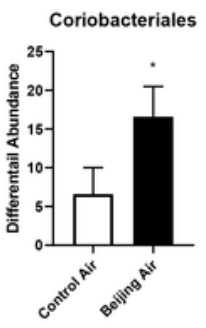

F

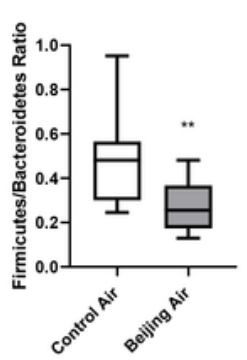

\section{Figure 8}

Luminal content microbiota analysis in male and female mice exposed in utero to control air or Beijinglike air ( $n=10 /$ group). A Chao1 a-diversity index. B Weighted UniFrac $\beta$-diversity index. C Overview of the relative abundance of gut bacteria depicted at the phylum level. D Overview of the relative abundance of gut bacteria depicted at the order level. E The differential abundance of significantly changed bacterial 
orders in female mice. ${ }^{\star} p<0.05,{ }^{\star} * \star p<0.005$ as determined by Corncob. F Firmicutes/Bacteroidetes ratio. ** $p<0.01$ as determined by the Mann-Whitney $U$ test.

\section{Supplementary Files}

This is a list of supplementary files associated with this preprint. Click to download.

- Additionallnformation.docx 